\title{
Effect of Repeated Injection of Iodixanol on Renal Function in Healthy Wistar Rats Using Functional MRI
}

\author{
Yongfang Wang, ${ }^{1}$ Ke Ren $\left(\mathbb{D},{ }^{1}\right.$ Lizhi Xie, ${ }^{2}$ Wenge Sun, ${ }^{1}$ Yi Liu, ${ }^{1}$ and Songbai Li ${ }^{1}$ \\ ${ }^{1}$ Department of Radiology, First Affiliated Hospital of China Medical University, Shenyang 110001, China \\ ${ }^{2}$ GE Healthcare, MR Research China, Beijing, China \\ Correspondence should be addressed to Ke Ren; renke815@sina.com
}

Received 12 July 2017; Revised 13 November 2017; Accepted 1 February 2018; Published 4 April 2018

Academic Editor: Eiichi Honda

Copyright (c) 2018 Yongfang Wang et al. This is an open access article distributed under the Creative Commons Attribution License, which permits unrestricted use, distribution, and reproduction in any medium, provided the original work is properly cited.

\begin{abstract}
Purpose. To determine the optimal time interval of repeated intravenous injections of iodixanol in rat model and to identify the injury location and causes of renal damage in vivo. Materials and Methods. Rats were randomly divided into Control group, Group 1 with one iodixanol injection, and Group 2 with two iodixanol injections. Group 2 was subdivided into 3 cohorts according to the interval between the first and second iodixanol injections as 1,3, and 5 days, respectively. Blood oxygen level-dependent (BOLD) imaging and diffusion weighted imaging (DWI) were performed at 1 hour, 1 day, 3 days, 5 days, and 10 days after the application of solutions. Results. Compared with Group $1(7.2 \%)$, Group 2 produced a remarkable R2* increment at the inner stripe of the renal outer medulla by $15.37 \%(P=0.012), 14.83 \%(P=0.046)$, and $13.53 \%(P>0.05)$, respectively, at 1 hour after repeated injection of iodixanol. The severity of BOLD MRI to detect renal hypoxia was consistent with the expression of HIF-1 $\alpha$ and R2* was well correlated with HIF-1 $\alpha$ expression $(r=0.704)$. The acute tubular injury was associated with urinary NGAL and increased significantly at 1 day. Conclusions. Repetitive injection of iodixanol within a short time window can induce acute kidney injury, the impact of which on renal damage in rats disappears gradually 3-5 days after the injections.
\end{abstract}

\section{Introduction}

Contrast agents (CMs) have been largely applied in clinical applications, which are particularly required upon the performance of various radiographic imaging modes and complex interventional procedures [1]. Clinicians encounter risk factors that may lead to Iodinated Contrast-Induced Acute Kidney Injury (CIAKI) [2]. For instance, the patients with recurrent diseases may be required to undergo repetitive injections of contrast agents during examinations. There is a general agreement that the administration of multiple CMs within a short period of time may put the patients at risk [3]. The Contrast Media Safety Committee (CMSC) regards this as a serious matter in clinical practice, and it is of significance to propose the optimal time interval between the procedures that require intravascular CMs injections [2]. To our best knowledge, there was no previous publishing regarding this health risk.

Serum creatinine ( $\mathrm{sCr}$ ) has been used as a major diagnostic criterion for contrast-induced renal impairment, which typically reaches its peak at $48-72 \mathrm{~h}$ after contrast medium exposure [4]. This suggests an intrinsic delay of therapy in patients who may have developed CIAKI, while it prolongs the duration of hospitalization for patients without CIAKI [5]. Recent studies have been focusing on identifying the early diagnostic biomarkers of AKI [6]. Neutrophil gelatinaseassociated lipocalin (NGAL) is proven to be one of the most significant biomarkers to detect CIAKI [7], which is observed to increase significantly prior to detectable changes in serum creatinine. Moreover, urinary NGAL (uNGAL) is demonstrated to reveal changes as early as $4 \mathrm{~h}$ after CM administration in rats [8] and $8 \mathrm{~h}$ in humans [9]. Therefore, uNGAL was applied as an indicator of renal injury after CMs in the present study.

The universally acknowledged causes of CIAKI include hypoxic and tubular damage as well as altered hemodynamics, despite the fact that not all the mechanisms leading to CIAKI have been revealed [10-12]. Renal hemodynamics and renal PO2 levels were investigated in Seeliger et al.s study by using an optical probe and laser-Doppler fluxmetry, whereas 
the invasiveness of such method prevents its application on patients [13]. Quantitative functional MRI (fMRI) has attracted considerable attention in recent years as a noninvasive approach to assess relative renal content [14, 15]. BOLD MRI has been shown to be competent in providing indirect measurements of real-time oxygenation status for renal damage provoked by $\mathrm{CM}$ with deeper understanding $[16,17]$. MR-based biomarker $\mathrm{T}^{*}$ is susceptible to changes in the volume of deoxygenated $\mathrm{Hb}(\operatorname{deoxyHb})$ per tissue volume element (voxel), and its reciprocal value $\left(\mathrm{R} 2{ }^{*}=1 / \mathrm{T} 2^{*}\right)$ has been explored in numerous studies to evaluate tissue hypoxia. Pedersen et al's study verified a linear relationship between the measurements of $\mathrm{R} 2{ }^{*}$ using BOLD imaging and the direct measurements of renal pO2 levels using oxygen sensitive microelectrodes in kidney [18]. However, recent report also showed that changes in $\mathrm{R} 2{ }^{*}$ are potentially confounded by compartmental volumes (tubular, vascular, and interstitial compartments) [19]. In addition, diffusion weighted imaging (DWI) based on the molecular diffusion of water is feasible in the assessment of renal functions, particularly in the detection of early stage renal failure caused by CIAKI [20]. DWI can be applied to measure the magnitude of Brownian water motion; moreover, apparent diffusion coefficient $(\mathrm{ADC})$ can quantitatively estimate water exchange rate between intra- and extracellular aspects of the kidney. Both techniques have been used extensively to assess the changes in renal physiology after CM injection. Preclinical studies were mostly conducted in a relatively short period of time, where little was published with regard to the changes of kidney function beyond 3 days after CM injection $[8,21]$. It was recently revealed that CM can induce long-term damage of kidney in the model with acute kidney injury [22, 23]. Thereafter, BOLD and DWI MRI were performed to examine contrast-induced renal impairment in iodixanol-treated rats over a relatively longer duration of time (i.e., 10 days) after duplicated injection.

In order to achieve an optimal time interval that protects the renal system in rats, repeated intravenous injections of iodixanol with different time intervals were performed to induce aggravate kidney damage in healthy Wistar rats, which may also reveal differences in causing potential kidney injury with respect to time, location, and intensity of potential damage. The current study also aimed to examine if renal function can gradually return to a level close to baseline at a later time point. Meanwhile, the study allowed us to monitor kidney damage by assessing sCr and uNGAL to evaluate renal parenchymal injury at the cellular level using histopathology and to detect the cause of renal injury with immunohistochemistry.

\section{Materials and Methods}

2.1. Subject Selection. The current animal study was approved by the institutional ethics committee of China Medical University and performed complied with our institute's Guidelines for the Care and Use of Laboratory Animals. Wistar male rats $300 \pm 20 \mathrm{~g}(n=85)$ were randomly divided into Group $1(n=17)$ with one iodixanol injection, Group $2(n=$ $51)$ with two injections, and the Control group $(n=17)$ with two saline injections separated by a 1-d interval. Group 2 was subdivided into 3 cohorts according to the interval between the first and second injections of iodixanol as 1-day, 3-day, and 5-day subgroups.

2.2. Experimental Protocols. Rats were allocated in metabolic cages for urine collections. They had free access to food and water until $8 \mathrm{~h}$ before MRI examination. They were anesthetized via an intraperitoneal infusion dose of $10 \%$ chloral hydrate at $0.3 \mathrm{~mL} / 100 \mathrm{~g}$. Urine samples were collected prior to BOLD and DWI MRI acquisition. After baseline fMRI scan, contrast or physiological saline was inserted in the tail vein. Iodixanol (Visipaque 320, GE Healthcare, Ireland) was prewarmed $\left(37^{\circ} \mathrm{C}\right)$ and uniformly injected as fast as possible. For Groups 1 and 2, intravenous contrast agent was administrated at a dosage of $4 \mathrm{~g}$ iodine $/ \mathrm{kg}$ body weight. In Group 1, fMRI was performed at $1 \mathrm{~h}, 1$ day, 3 days, 5 days, and 10 days after the injection of iodixanol. Group 2 underwent a second iodixanol injection at a respective interval of 1 , 3 , and 5 days after the first injection. The fMRI scan time points were $1 \mathrm{~h}, 1$ day, 3 days, 5 days, and 10 days after the second application of iodixanol. After each fMRI acquisition, three rats were randomly selected and given an overdose of anesthetic; thereafter, the kidneys were immediately removed and the urine samples were collected at each respective time point. Physiological saline was used as placebo in place of iodixanol in Control group for the same scan (Figure 1).

2.3. MRI Protocol and Data Analysis. After $30 \mathrm{~min}$ of anesthesia, all MRI acquisitions were performed on a 3.0 T Twin Speed whole-body MR scanner (General Electric Medical Systems, Milwaukee, WI, USA) with a small extremity coil. In order to minimize the artifacts induced by bowel loops susceptibility, all the subjects were placed in the right decubitus position with their kidneys at the center of the rat array coil. BOLD and DWI images were independently acquired by 2 professional radiologists in a double-blinded manner. Data acquisition parameters are demonstrated in Table 1.

Parametric images of $\mathrm{R} 2{ }^{*}$ and $\mathrm{ADC}$ were analyzed on ADVANCE 4.6 Workstation software (General Electric Medical Systems). According to the study of Li et al. [8, 24], all layers of renal tissues and the corresponding $\mathrm{R} 2^{*}$ weighted image were segmented into four regions, including cortex (CO), outer stripe of the outer medulla (OSOM), inner stripe of the outer medulla (ISOM), and inner medulla (IM) (Figures 2(a) and 2(b)). In each kidney, a single region of interest (ROI) corresponding to the histological sections was placed by a manual segmentation of the coronal BOLD images and DWI images. Moreover, the quantitative regional R2* and ADC measurements were performed. ROI was marked as large as possible $\left(>18 \mathrm{~mm}^{2}\right)$ (Figure $2(\mathrm{c})$ ).

2.4. Pathology and Immunohistochemistry. In each group, three rats were sacrificed for histological studies at specific time points ( $1 \mathrm{~h}, 1 \mathrm{~d}, 3 \mathrm{~d}, 5 \mathrm{~d}$, and $10 \mathrm{~d}$ ). Fixation of kidneys with $4 \%$ paraformaldehyde for $72 \mathrm{~h}$, dehydration, paraffin embedding, and sectioning $(5-\mu \mathrm{m})$ were performed for Hematoxylin-Eosin (H\&E) staining. Two clinical pathologists with more than 5 years of experience independently 


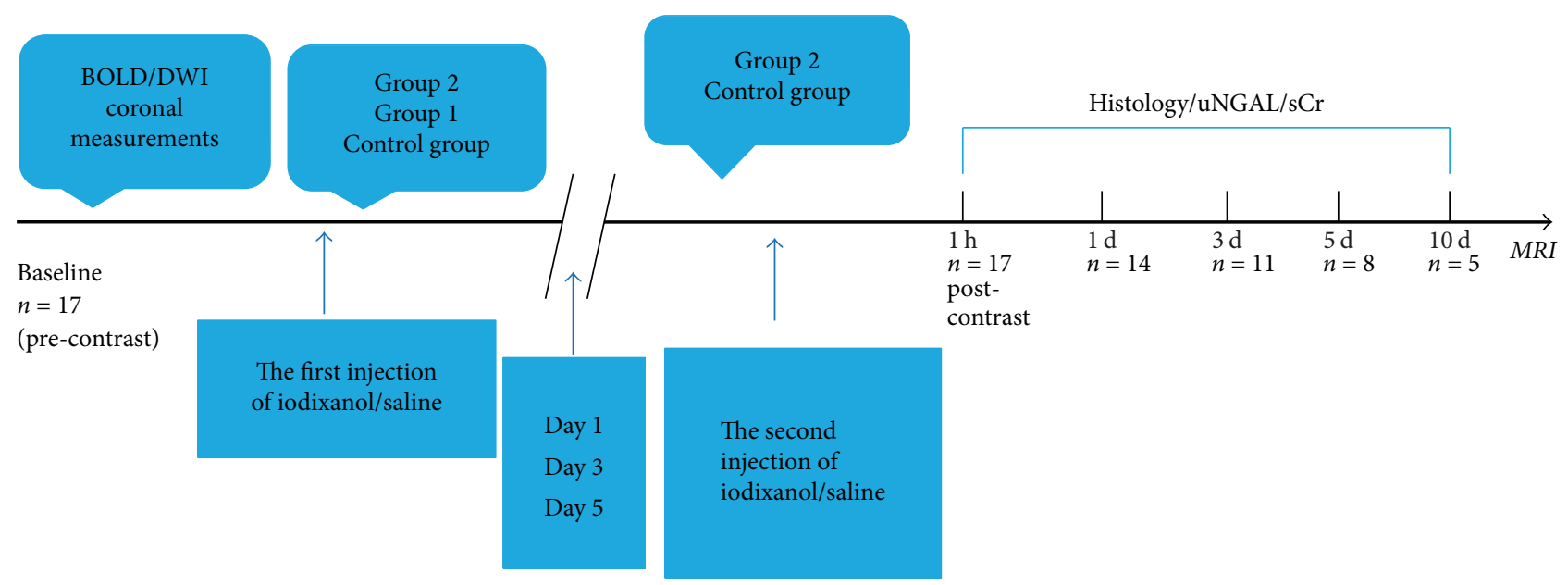

FIGURE 1: The experimental flow chart. This flowchart illustrates the specific measurement protocol and the time points of fMRI, histology, uNGAL, and sCr that occurred.

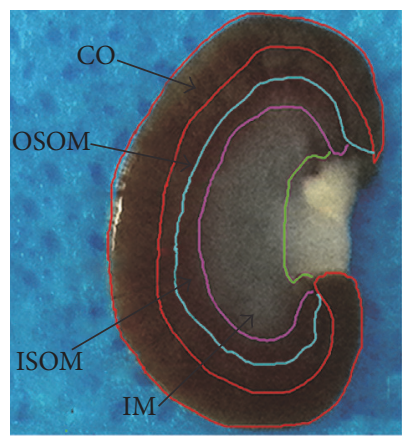

(a)

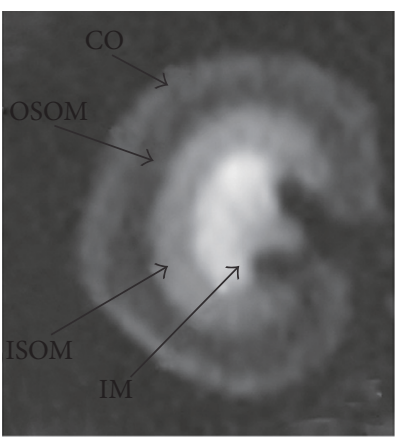

(b)

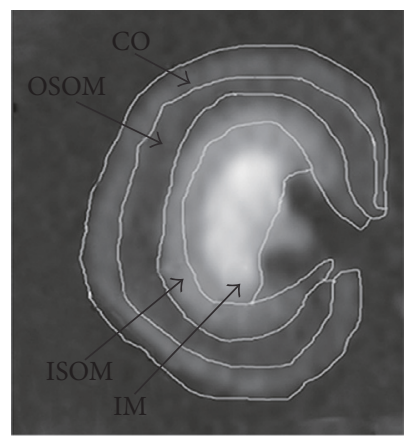

(c)

FIgURE 2: The four kidney areas corresponding to anatomic image. (a) Resected specimen. (b) T2 image. (c) The ROIs.

TABLE 1: fMRI parameters applied in BOLD and DWI acquisitions.

\begin{tabular}{lcc}
\hline & BOLD & DWI \\
\hline Number of slices & 3 & 3 \\
Section thickness, mm & 2.4 & 2.4 \\
Repetition time, ms & $5.4-11$ & 4500 \\
Echo time, ms & Coronal & 106.9 \\
Orientation & 41.67 & $62.5-250$ \\
Bandwidth, hertz per pixel & $100 \times 100$ & $100 \times 100$ \\
Field of view, mm & $256 \times 256$ & $160 \times 160$ \\
Matrix & 2 & 8 \\
Number of excitations & 2 min $5 \mathrm{~s}$ & $1 \mathrm{~min} 57 \mathrm{~s}$ \\
Acquisition time & - & 0,600 \\
$b$-values (s/mm ${ }^{2}$ ) & $30^{\circ}$ & $90^{\circ}$ \\
Flip angle & Free breathing & Free breathing \\
Breathing protocol & & 160 \\
\hline
\end{tabular}


examined the sections. At a magnification of 200x, intracytoplasmic vacuoles were revealed in the cortex, predominantly in the proximal convoluted tubules. Five different fields were randomly selected within each slide at each time point. The kidney was analyzed according to size and number of vacuoles, pronounced tubular dilatation, and interstitial vasodilation and congestion [25]. The severity of tubular injury was semiquantitatively analyzed with a scale of 0-4 assigned to each histopathological change, where 0 is no damage; 1 , minimal injury (less than 5\%); 2, moderate injury (between $5 \%$ and $25 \%$ ); 3 , intermediate injury (between $25 \%$ and $75 \%$ ); and 4, severe injury (more than 75\%) [26]. Hypoxia-inducible factor- $1 \alpha$ staining could assess the degree of hypoxia in the intrarenal tissues for different groups. We evaluated renal parenchymal hypoxia semiquantitatively based on the degree of HIF- $1 \alpha$ staining in the CO, OSOM, and ISOM, as well as IM. Grading was performed in a blinded manner. At 400x magnification, 5 randomly selected areas of each rat were scored according to both signal intensity and abundance [27], where 0 is no expression; 1 , expression of $<25 \%$ of the examination field; 2 , expression of $26 \%$ to $50 \%$; 3 , expression of $51 \%$ to $75 \%$; and 4 , expression of $>75 \%$ of the examination field; for signal intensity, 1 is moderate and 2 is strong.

Sections were pretreated in the same way as described above. Then, after deparaffinization, antigen retrieval, and peroxidase quenching, the samples were blocked with 5\% normal goat serum and then incubated with anti-HIF-1 $\alpha$ antibody (dilution 1:200) (Abcam, ab2185, Cambridge, MA, USA) overnight at $4^{\circ} \mathrm{C}$. Subsequently, the tissue sections were incubated with the biotinylated anti-rabbit IgG secondary antibody (dilution 1:200) (A0277, Beyotime, Shanghai, China) for $1 \mathrm{~h}$ at room temperature.

2.5. Urinary Biomarker NGAL. In each group, after the injection of iodixanol or saline, 3 urine samples $(0.5-2.0 \mathrm{~mL})$ were collected at $1 \mathrm{~h}$ and $1,3,5$, and 10 days. The samples were centrifuged at $3,200 \mathrm{rpm}\left(4^{\circ} \mathrm{C}\right)$ for 20 minutes and subsequently placed into a $-80^{\circ} \mathrm{F}$ freezer for storage. The concentration of urinary NGAL was analyzed using ELISA assays from Abcam (Cambridge, MA, USA), following the standard protocols. In order to minimize any confounding effects of urine flow rate, concentration levels of NGAL were normalized to urine creatinine concentrations (analyzed in local clinical laboratory) [28].

2.6. Serum Creatinine Assessment. To further verify the injury of kidney and the severity of the disease, blood serum creatinine was measured. The samples were collected from the venae angularis of the rats and were centrifuged at a speed of 3,500 rpm $\left(4^{\circ} \mathrm{C}\right)$ for 20 minutes. The blood serum creatinine concentrations were analyzed in local clinical laboratory.

2.7. Statistical Analysis. SPSS22.0 (SPSS Inc., Chicago, IL, USA) was used for statistical analyses, where $P$ value $<0.05$ was considered as statistically significant. All the data were tested for normality. One way analysis of variance (ANOVA) was followed by LSD method or Kruskal-Wallis test (nonnormal distributions) to compare $\mathrm{R} 2{ }^{*} / \mathrm{ADC}$ values across cohorts at the same time points. FMRI data in each group were compared with the baseline value using repeated ANOVA test or Bonferroni post hoc test. Spearman's correlation analysis was employed to assess the relationship between BOLD parameters and pathological variables.

\section{Results}

3.1. BOLD-Image Postprocessing Results. The spatial resolution of R2* images was capable enough of distinguishing $\mathrm{CO}$, OSOM, ISOM, and IM of the kidney (Figure 3 ). R2* values showed the least amount of changes in the Control group with respect to the time course, which confirmed the stability of BOLD MRI during the acquisition period.

The montage image of $\mathrm{R} 2 *$ in the kidney of a representative rat was demonstrated in respect to time course over a 10-day period after iodixanol injection using the BOLD sequence. After repeated injection of iodixanol, R2* values increased significantly over time in the 1-day subgroup, where renal ISOM showed the largest differences in these tissues, and the duration of $\mathrm{R} 2{ }^{*}$ ( 5 days) in ISOM was longer than that of other regions. ISOM demonstrated higher intensity of signals than that of CO in BOLD images, which probably indicated that oxygenation status was lower in ISOM. In OSOM, R2* values were more significant, while the duration (3 days) was shorter than that of ISOM. R2* values in IM showed a fast rise in the reduplicated-treated rats, followed by a return to baseline on day 3 . In renal cortex, $\mathrm{R} 2{ }^{*}$ values were continuously higher compared with baseline values at the time points ( $1 \mathrm{~h}$ to 5 days; all $P<0.05)$. A nearly complete recovery of baseline renal function was observed within 10 days in the 3-day subgroup, while Group 1 required 5 days to return to the baseline levels in all compartments. Figure 4(a) presents that $\mathrm{R} 2^{*}$ values gradually recovered towards the baseline during the entire period of study in all 5 groups.

The average change of hypoxia levels in kidneys in each group was summarized in Table 2. Iodixanol increased R2* values to the maximum levels at $1 \mathrm{~h}$, which was in accordance with previous animal studies $[16,29]$. Compared with Group $1(7.2 \%)$, Group 2 produced a remarkable $\mathrm{R} 2{ }^{*}$ increment at inner stripe of the renal outer medulla by $15.37 \%(P=0.012)$, 14.83\% $(P=0.046)$, and $13.53 \%(P>0.05)$, respectively, at 1 hour after repeated injection of iodixanol. These results indicated that short-term repeated injection of iodixanol is a risk factor for CIAKI, while 3-5-day interval is the optimal time interval for a second injection.

3.2. DWI-Image Postprocessing Results. ADC values gradually recovered towards the baseline over time in all the groups (Figure 4(b)). In all the renal regions, ADC values showed a similar rapid initial decrease, reaching the bottom at $1 \mathrm{~h}$ after iodixanol administration, followed by a rebound towards the baseline (Figure 5). Among these 5 groups, the 1-day subgroup exhibited the greatest depression of ADC compared with the remaining groups. For each renal region (CO, OSOM, ISOM, and IM), a significant decrease in ADC lasted for 3 days in $\mathrm{CO}(P=0.037)$ and OSOM $(P=$ $0.005)$, as well as 5 days in $\operatorname{ISOM}(P=0.008)$ in the 1 -day subgroup. In IM, ADC values showed the strongest decline at 

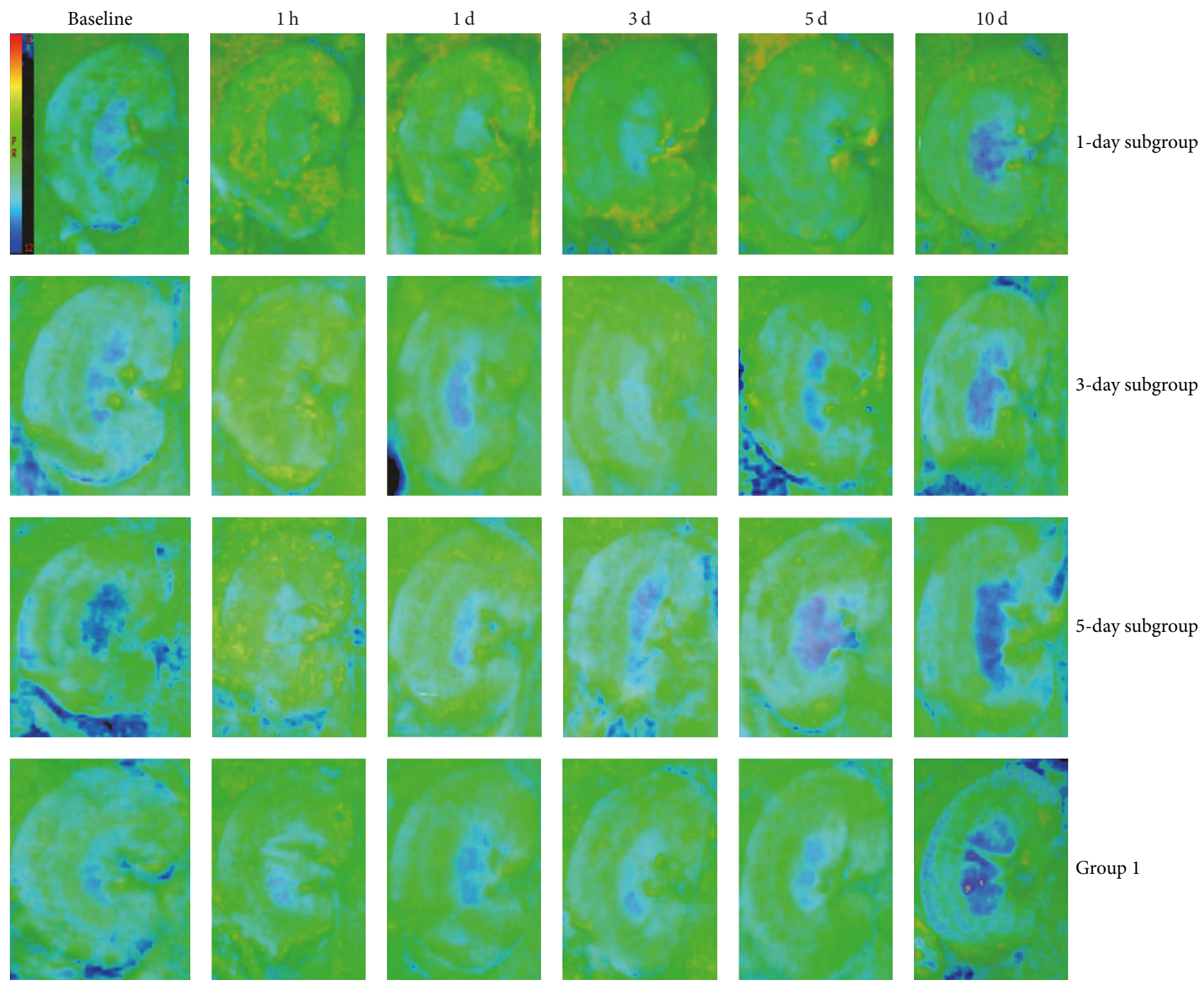

5-day subgroup
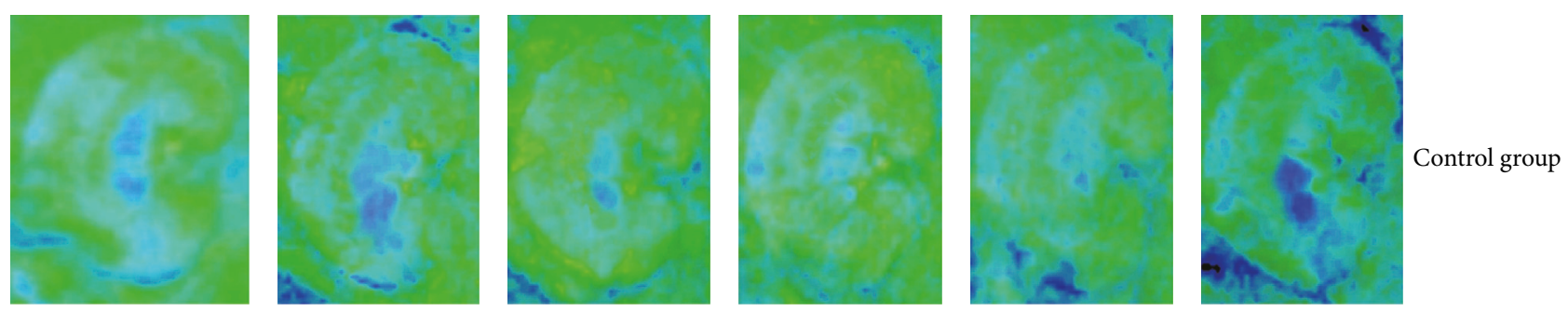

Figure 3: Representative $R 2^{*}$ maps (baseline, $1 \mathrm{~h}, 1$ day, 3 days, 5 days, and 10 days). All maps are demonstrated on the same window and level settings. 1-day, 3-day, and 5-day subgroups were injected for a second time at different time intervals. Group 1 was injected with iodixanol only once. The intensity of ISOM was larger than the remaining regions, which implies lower level of oxygenation.

$1 \mathrm{~h}(P=0.003)$ but recovered to the baseline level after the first day. At the subsequent time points, ADC values in the 3 -day subgroup gradually reached a level close to baseline at day 5 in all areas, while the 5 -day subgroup recovered to the baseline level after 3 days. ADC values increased slightly for the rats injected with saline at $1 \mathrm{~h}$, but they did not produce statistical significance for all the time points. At 1-hour time point, compared with that of Group 1, ADC values were significantly reduced by 1 -day subgroup, 3 -day subgroup, and 5-day subgroup in CO (all $P<0.001)$, OSOM $(P<0.001$, $P=0.017, P>0.05$, resp.), ISOM $(P=0.038, P>0.05$, $P>0.05$, resp.), and IM (all $P<0.001$ ). Table 3 summarized the time course for ADC values in Group 2.

3.3. Histologic Analysis. A high incidence of vacuole formation occurred in all the subjects after the injection of iodixanol, especially in the repeated injection groups. In Group 2, the maximum modifications of vacuoles appeared 

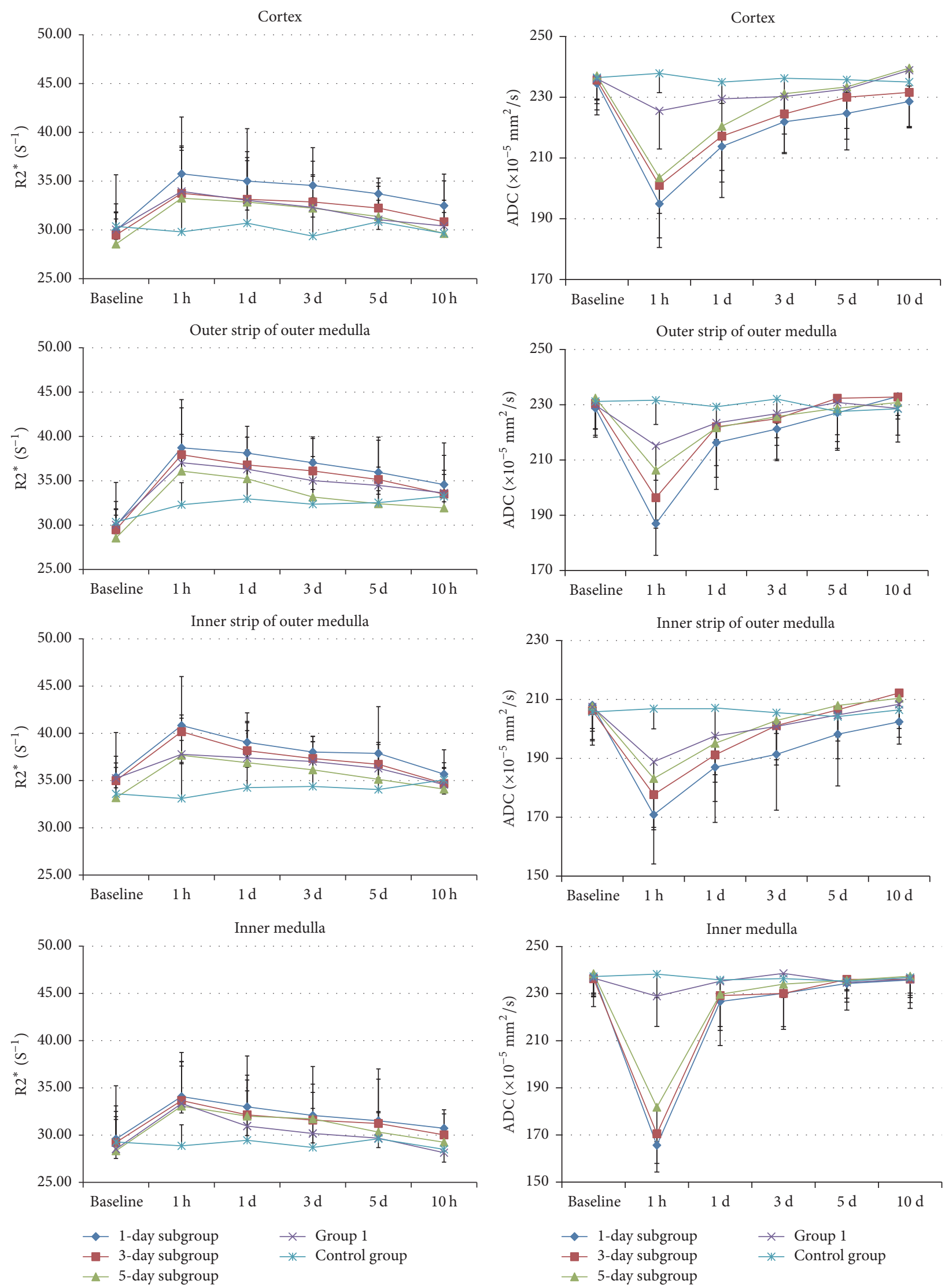

(a)

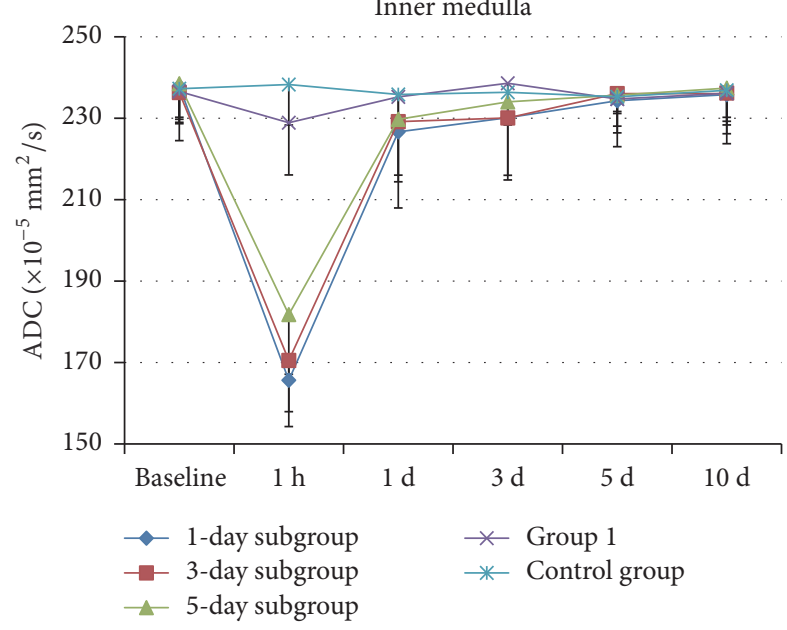

(b)

FIGURE 4: Summary of the temporal changes in R2*/ADC measurement during a long period of time. (a) R2 ${ }^{*}$ time curves. (b) ADC time curves. Notice maximum $\mathrm{R}^{*} /$ minimum $\mathrm{ADC}$ values appeared at $1 \mathrm{~h}$, followed by gradual regression to a near-complete baseline level over time. 
Baseline
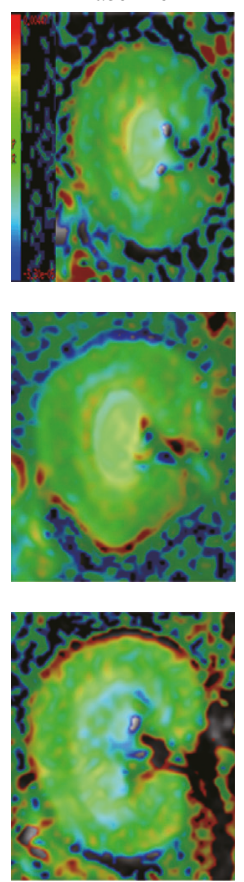

$1 \mathrm{~h}$
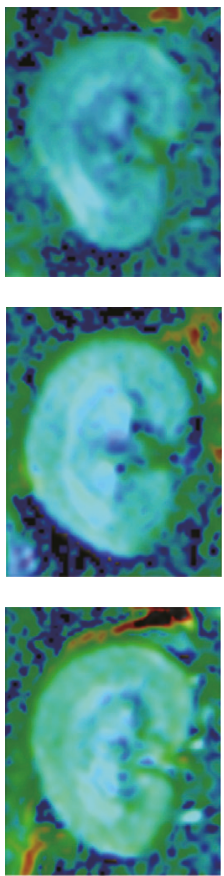

$1 \mathrm{~d}$
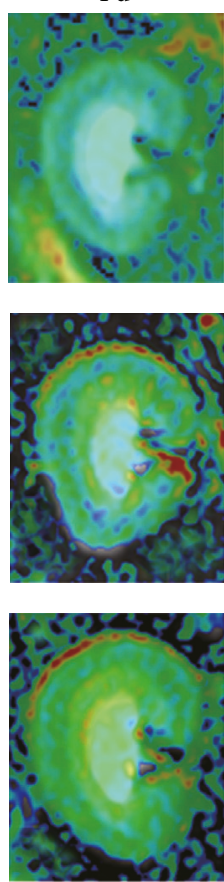

$3 d$
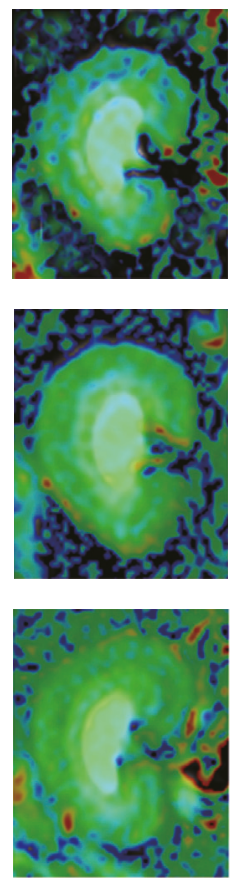

$5 \mathrm{~d}$
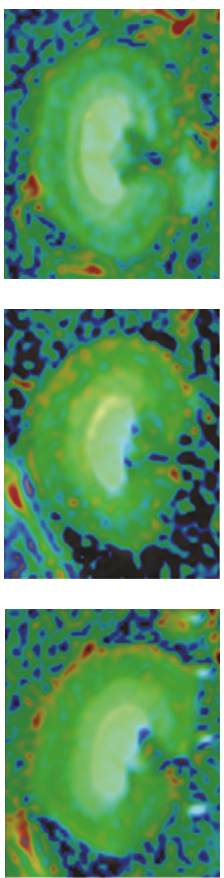

$10 \mathrm{~d}$
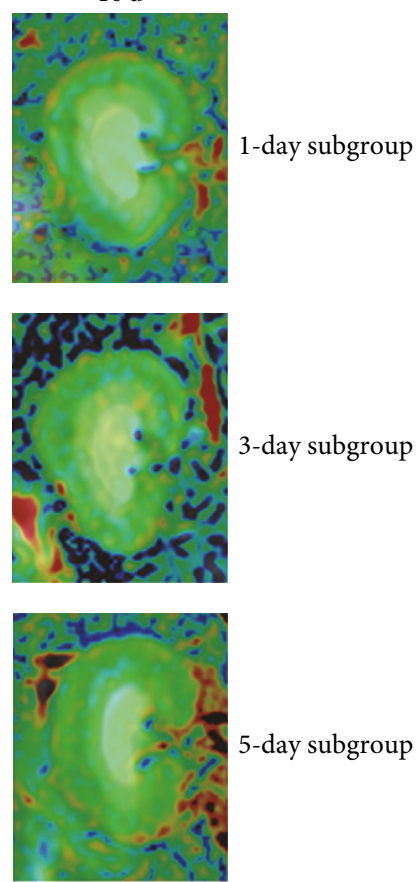

Figure 5: Representative ADC maps (baseline, $1 \mathrm{~h}, 1$ day, 3 days, 5 days, and 10 days). The cortical and medullary ADC in the kidney decreased in Group 2 after iodixanol injection, especially in 1-day subgroup. For each renal region, they significantly decreased at $1 \mathrm{~h}$ and thereafter gradually increased upward towards the baseline.

TABLE 2: R2* values changes in 4 renal regions in all groups over time $\left(\mathrm{s}^{-1}\right)$.

\begin{tabular}{|c|c|c|c|c|c|c|c|}
\hline Kidney tissue & Treatment & $\begin{array}{l}\text { Baseline } \\
(n=17)\end{array}$ & $\begin{array}{c}1 \mathrm{~h} \\
(n=17)\end{array}$ & $\begin{array}{c}1 \mathrm{~d} \\
(n=14)\end{array}$ & $\begin{array}{c}3 \mathrm{~d} \\
(n=11)\end{array}$ & $\begin{array}{c}5 \mathrm{~d} \\
(n=8)\end{array}$ & $\begin{array}{c}10 \mathrm{~d} \\
(n=5)\end{array}$ \\
\hline \multirow{5}{*}{$\mathrm{CO}$} & 1-day subgroup & $29.95 \pm 2.01$ & $35.75 \pm 5.70^{*}$ & $35.00 \pm 5.82^{*}$ & $34.56 \pm 5.38^{*}$ & $33.70 \pm 3.88^{*}$ & $32.48 \pm 1.61$ \\
\hline & 3-day subgroup & $29.48 \pm 1.64$ & $33.75 \pm 4.70^{*}$ & $33.14 \pm 4.28^{*}$ & $32.86 \pm 4.18^{*}$ & $32.24 \pm 2.58^{*}$ & $30.83 \pm 4.20$ \\
\hline & 5-day subgroup & $28.55 \pm 3.21$ & $33.25 \pm 4.91^{*}$ & $32.85 \pm 5.17^{*}$ & $32.23 \pm 3.42^{*}$ & $31.36 \pm 3.10$ & $29.63 \pm 3.41$ \\
\hline & Group 1 & $30.03 \pm 1.82$ & $33.95 \pm 4.66^{*}$ & $33.03 \pm 4.07^{*}$ & $32.31 \pm 3.18^{*}$ & $31.05 \pm 1.03$ & $30.40 \pm 1.38$ \\
\hline & Control group & $30.36 \pm 2.31$ & $29.79 \pm 3.61$ & $30.69 \pm 2.72$ & $29.36 \pm 3.94$ & $30.83 \pm 2.20$ & $29.65 \pm 6.07$ \\
\hline \multirow{5}{*}{ OSOM } & 1-day subgroup & $33.53 \pm 3.06$ & $38.72 \pm 4.87^{*}$ & $38.12 \pm 4.50^{*}$ & $37.04 \pm 3.02^{*}$ & $35.95 \pm 2.91$ & $34.57 \pm 3.62$ \\
\hline & 3-day subgroup & $33.14 \pm 2.36$ & $37.94 \pm 6.22^{*}$ & $36.79 \pm 3.12^{*}$ & $36.11 \pm 1.63^{*}$ & $35.14 \pm 4.78$ & $33.50 \pm 5.77$ \\
\hline & 5-day subgroup & $31.86 \pm 4.40$ & $36.09 \pm 1.80^{*}$ & $35.23 \pm 1.90^{*}$ & $33.17 \pm 4.21$ & $32.41 \pm 1.47$ & $31.94 \pm 4.25$ \\
\hline & Group 1 & $34.28 \pm 2.69$ & $37.04 \pm 3.20^{*}$ & $36.31 \pm 2.17^{*}$ & $35.02 \pm 4.75$ & $34.48 \pm 2.06$ & $33.63 \pm 4.24$ \\
\hline & Control group & $31.72 \pm 5.62$ & $32.30 \pm 2.48$ & $32.97 \pm 3.50$ & $32.36 \pm 2.65$ & $32.54 \pm 1.94$ & $33.25 \pm 2.46$ \\
\hline \multirow{5}{*}{ ISOM } & 1-day subgroup & $35.39 \pm 3.55$ & $40.83 \pm 4.70^{* \dagger}$ & $39.05 \pm 5.18^{*}$ & $38.02 \pm 2.21^{*}$ & $37.87 \pm 1.09^{*}$ & $35.68 \pm 4.95$ \\
\hline & 3-day subgroup & $34.99 \pm 2.59$ & $40.18 \pm 1.43^{* \dagger}$ & $38.17 \pm 4.00^{*}$ & $37.33 \pm 1.80^{*}$ & $36.73 \pm 1.10^{*}$ & $34.68 \pm 3.58$ \\
\hline & 5-day subgroup & $33.19 \pm 1.89$ & $37.68 \pm 4.27^{*}$ & $36.90 \pm 4.22^{*}$ & $36.14 \pm 3.53^{*}$ & $35.13 \pm 2.99$ & $34.10 \pm 2.20$ \\
\hline & Group 1 & $35.24 \pm 1.63$ & $37.78 \pm 1.47^{*}$ & $37.40 \pm 2.89^{*}$ & $37.02 \pm 2.68^{*}$ & $36.29 \pm 2.75$ & $34.57 \pm 1.81$ \\
\hline & Control group & $33.60 \pm 2.79$ & $33.11 \pm 3.82$ & $34.25 \pm 4.12$ & $34.32 \pm 3.91$ & $34.05 \pm 4.77$ & $35.07 \pm 1.80$ \\
\hline \multirow{5}{*}{ IM } & 1-day subgroup & $29.59 \pm 3.60$ & $34.08 \pm 5.64^{*}$ & $32.99 \pm 3.23^{*}$ & $32.08 \pm 2.84$ & $31.52 \pm 2.44$ & $30.72 \pm 4.40$ \\
\hline & 3-day subgroup & $29.23 \pm 3.87$ & $33.66 \pm 5.09^{*}$ & $32.15 \pm 4.19$ & $31.59 \pm 3.81$ & $31.24 \pm 5.77$ & $30.03 \pm 2.65$ \\
\hline & 5-day subgroup & $28.34 \pm 3.62$ & $33.07 \pm 4.69^{*}$ & $32.01 \pm 6.36$ & $31.72 \pm 5.54$ & $30.32 \pm 2.18$ & $29.25 \pm 3.00$ \\
\hline & Group 1 & $28.53 \pm 3.13$ & $33.34 \pm 4.45^{*}$ & $30.96 \pm 3.73$ & $30.17 \pm 2.66$ & $29.68 \pm 1.35$ & $28.14 \pm 2.57$ \\
\hline & Control group & $29.27 \pm 3.24$ & $28.87 \pm 2.23$ & $29.45 \pm 3.60$ & $28.69 \pm 2.66$ & $29.61 \pm 2.75$ & $28.49 \pm 2.21$ \\
\hline
\end{tabular}

${ }^{*} P<0.05$ versus baseline; ${ }^{\dagger} P<0.05$ versus Group 1 . 
TABLE 3: ADC values changes in the 4 renal regions in Group $2\left(\times 10^{-5} \mathrm{~mm}^{2} / \mathrm{s}\right)$.

\begin{tabular}{llcccccc}
\hline Kidney tissue & Treatment & $\begin{array}{c}\text { Baseline } \\
(n=17)\end{array}$ & $\begin{array}{c}1 \mathrm{~h} \\
(n=17)\end{array}$ & $\begin{array}{c}1 \mathrm{~d} \\
(n=14)\end{array}$ & $\begin{array}{c}3 \mathrm{~d} \\
(n=11)\end{array}$ & $\begin{array}{c}5 \mathrm{~d} \\
(n=8)\end{array}$ & $\begin{array}{c}10 \mathrm{~d} \\
(n=5)\end{array}$ \\
\hline \multirow{3}{*}{ CO } & 1-day subgroup & $235 \pm 10$ & $195 \pm 14^{*}$ & $214 \pm 17^{*}$ & $222 \pm 10^{*}$ & $225 \pm 12$ & $229 \pm 8$ \\
& 3-day subgroup & $236 \pm 8$ & $201 \pm 17^{*}$ & $217 \pm 15^{*}$ & $225 \pm 13^{*}$ & $230 \pm 14$ & $232 \pm 11$ \\
& 5-day subgroup & $237 \pm 8$ & $204 \pm 12^{*}$ & $221 \pm 15^{*}$ & $231 \pm 8$ & $233 \pm 9$ & $240 \pm 6$ \\
\hline \multirow{3}{*}{ OSOM } & 1-day subgroup & $229 \pm 8$ & $187 \pm 11^{*}$ & $216 \pm 17^{*}$ & $221 \pm 11^{*}$ & $227 \pm 10$ & $233 \pm 7$ \\
& 3-day subgroup & $231 \pm 9$ & $196 \pm 11^{*}$ & $222 \pm 14$ & $225 \pm 7$ & $232 \pm 5$ & $233 \pm 5$ \\
& 5-day subgroup & $232 \pm 11$ & $206 \pm 18^{*}$ & $222 \pm 18$ & $226 \pm 16$ & $229 \pm 15$ & $231 \pm 6$ \\
\hline \multirow{3}{*}{ ISOM } & 1-day subgroup & $208 \pm 9$ & $171 \pm 17^{*}$ & $187 \pm 19^{*}$ & $191 \pm 19^{*}$ & $198 \pm 18^{*}$ & $202 \pm 5$ \\
& 3-day subgroup & $206 \pm 10$ & $178 \pm 12^{*}$ & $191 \pm 16^{*}$ & $201 \pm 12$ & $206 \pm 11$ & $212 \pm 6$ \\
& 5-day subgroup & $208 \pm 8$ & $183 \pm 17^{*}$ & $195 \pm 13^{*}$ & $203 \pm 12$ & $208 \pm 11$ & $210 \pm 10$ \\
\hline \multirow{2}{*}{ IM } & 1-day subgroup & $238 \pm 9$ & $166 \pm 11^{*}$ & $227 \pm 19$ & $230 \pm 14$ & $234 \pm 6$ & $236 \pm 10$ \\
& 3-day subgroup & $236 \pm 7$ & $171 \pm 13^{*}$ & $229 \pm 13$ & $230 \pm 15$ & $236 \pm 5$ & $236 \pm 6$ \\
& 5-day subgroup & $238 \pm 8$ & $182 \pm 15^{*}$ & $230 \pm 15$ & $234 \pm 6$ & $236 \pm 4$ & $237 \pm 9$ \\
\hline
\end{tabular}

DWI measurements were performed at baseline and $1 \mathrm{~h}, 1$ day, 3 days, 5 days, and 10 days after the second injection, and mean ADC of each region of interest was measured. ${ }^{*} P<0.05$ versus baseline.

at $1 \mathrm{~h}$; on day 1 , tubular epithelial cells swelled up and broke down; on day 3 , pronounced tubular dilatation and mild vacuoles formation were observed after the intravenous repeated injection of iodixanol; on day 5, the glomeruli gradually became atrophy and fibrosis, and the proximal and distal convoluted tubule epithelial cells were cloudy and swollen; finally, on day 10, the glomeruli exhibited overt atrophy and fibrosis, and a small amount of interstitial vasodilation was revealed (Figure 6).

At 1 hour, the magnitude and severity of renal tubular damage depended on the frequency and interval of injections. Compared with Group 1, the highest severity was observed after the repeated injection with a short interval of only $1-3 \mathrm{~d}$ in OSOM $(P=0.031, P=0.049$, resp. $)$ and in ISOM $(P=$ $0.031, P=0.048$, resp.); in addition, the least severe tubules injury was present after the treatment with iodixanol for both OSOM and ISOM in the 5-day subgroup $(P>0.05, P>0.05$, resp.) (Figure 7).

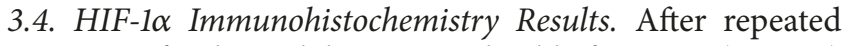
injection of iodixanol, hypoxia-inducible factor- $1 \alpha$ (HIF-1 $\alpha$ ) was transiently upregulated at $1 \mathrm{~h}$, the expression of which was most significant in ISOM area. HIF immunostaining was confined to a short period of time, within 5 days after the induction of hypoxic insult in Group 2 (Figure 8). 3-day and 5 -day subgroups showed less expression of HIF-1 $\alpha$ than 1day subgroup at 1 hour. At 1 hour, compared with Group 1, immunostaining of renal HIF-1 $\alpha$ was aggravated in Group 2 after the second injection of iodixanol in ISOM $(P=0.008$, $P=0.044, P>0.05$, resp.).

3.5. Urinary Neutrophil Gelatinase-Associated Lipocalin. CIAKI was confirmed to decrease in renal function after iodixanol injection using urinary neutrophil gelatinaseassociated lipocalin (uNGAL) as a marker. As shown in Figure 9, the statistically significant change of uNGAL was observed after iodixanol injection within 5 days for Group 2.
3.6. Serum Creatinine Results. As shown in Table 4, the sCr concentration reached its maximum value on day 3 in 1-day subgroup and 3 -day subgroup $(P=0.036, P=0.035$ versus baseline, resp.), whereas the difference was not statistically significant at other time points in all groups $(P>0.05$ versus baseline).

3.7. Correlation between BOLD Parameters and Renal Injury and HIF-1 $\alpha$ Expression Scores. As shown in Figure 10, the correlation between BOLD parameters and pathological scores, HIF- $1 \alpha$ expression scores of inner stripe of outer medulla, was decided by linear regression analysis. There was a good correlation between R2 ${ }^{*}$ and HIF- $1 \alpha$ expression $(P<0.0001$; $r=0.704)$; and $\mathrm{R} 2{ }^{*}$ was found to be well correlated with pathological scores $(P<0.0001 ; r=0.625)$. Meanwhile, there was fair correlation between histological scores and urine NGAL $(P<0.0001 ; r=0.520)$.

\section{Discussion}

Plenty of guidelines on CIAKI proposed to avoid multiple injections with CMs on patients especially within 48-72 hours after the first injection [2,30]. However, optimization of the clinical interval is of significance for the supervision of such treatments and prevention of the occurrence of CIAKI in clinical practice. Additionally, iodixanol has been widely observed in recent studies due to its iso-osmolality with plasma [31]. Therefore, the current study is a preliminary assessment to the impact of repetitive iodixanol injections on the damage sites in order to optimize the interval of repeated iodixanol administration.

The mechanisms leading to direct CIAKI have not been fully understood. However, the previously revealed potential risk factors act in a time and concentration dependent manner, indicating that the duplicate injection of CM in a short-term duration of time may aggravate acute damage of kidney [2]. In the current study, a detected increase in R2* 

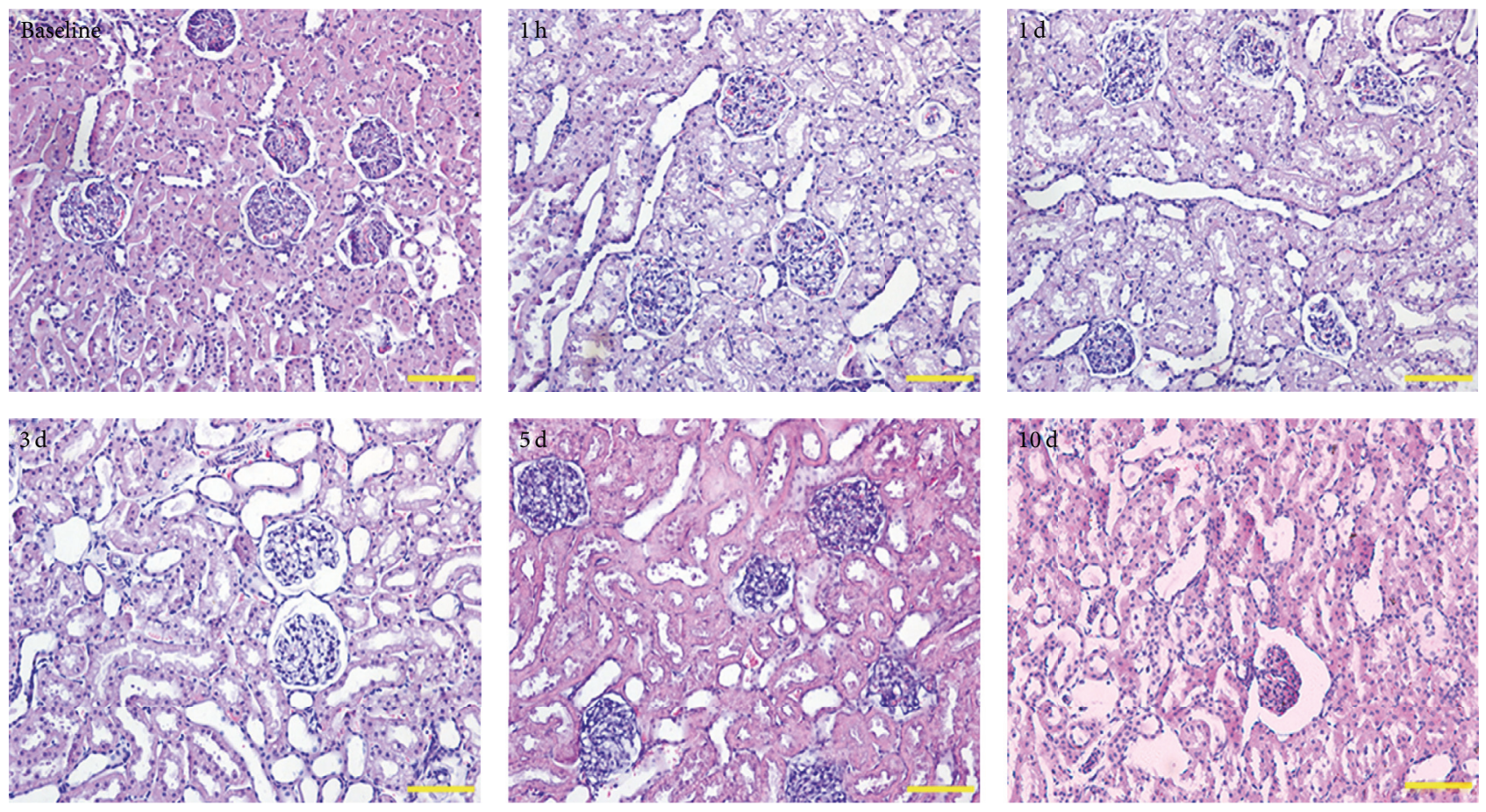

Figure 6: Hematoxylin-Eosin (H\&E) stained histological sections of the kidneys in the cortex. Renal histological injury subjected to repeated injection of iodixanol at different time points in 1-day subgroup. Scale bar, $100 \mu \mathrm{m}$.

TABLE 4: The time course of serum creatinine (umol/L) in all the iodixanol-treated groups.

\begin{tabular}{lcccccc}
\hline Groups & $\begin{array}{c}\text { Baseline } \\
(n=3)\end{array}$ & $\begin{array}{c}1 \mathrm{~h} \\
(n=3)\end{array}$ & $\begin{array}{c}1 \mathrm{~d} \\
(n=3)\end{array}$ & $\begin{array}{c}3 \mathrm{~d} \\
(n=3)\end{array}$ & $\begin{array}{c}5 \mathrm{~d} \\
(n=3)\end{array}$ & $\begin{array}{c}10 \mathrm{~d} \\
(n=3)\end{array}$ \\
\hline 1-day subgroup & $28.43 \pm 3.12$ & $31.97 \pm 3.58$ & $35.01 \pm 4.38$ & $43.18 \pm 3.24^{*}$ & $37.26 \pm 2.31$ & $32.85 \pm 5.04$ \\
3-day subgroup & $27.59 \pm 2.49$ & $30.47 \pm 3.53$ & $33.08 \pm 5.27$ & $39.12 \pm 2.46^{*}$ & $34.41 \pm 4.08$ & $28.22 \pm 2.04$ \\
5-day subgroup & $28.12 \pm 3.29$ & $30.09 \pm 3.93$ & $34.24 \pm 3.00$ & $36.13 \pm 3.73$ & $34.50 \pm 2.11$ & $27.46 \pm 2.30$ \\
Group 1 & $27.82 \pm 3.54$ & $29.33 \pm 3.18$ & $32.05 \pm 1.68$ & $33.96 \pm 2.07$ & $30.02 \pm 3.68$ & $27.59 \pm 2.67$ \\
\hline
\end{tabular}

${ }^{*} P<0.05$ versus baseline.

after CM injections in Group 2 may be associated with the occurrence of CIAKI as determined by urinary NGAL. The escalated distribution of HIF- $1 \alpha$ expression in renal tissues highlighted the degree of cellular hypoxia and a subsequent transcriptional response in CIAKI. Our results demonstrated a significant increased risk of CIAKI among the subjects that received a second dose of $\mathrm{CM}$ within one day after their first injection. Litter aggravate renal damage was observed in 3day or 5-day subgroups. Moreover, a decreased expression of HIF- $1 \alpha$ was observed in rats with the second injection on 3 or 5 days after their previous injection, which suggested a longer interval time between two CM injections. The possible mechanism may relate to cytotoxic effect, since iodixanol cytotoxicity is mostly ( $25 \%)$ observed within 15 minutes, to the maximum at 3 hours [32]. Taken together, it was reported that $70-85 \%$ of the injected contrast agent was cleared within 24 hours in humans [33], which reinforced the statement that even a short period of repeated exposure could activate the cascade leading to kidney damage but mostly the cumulative effect of that initial exposure vanished in the later time periods (after 5 days).

There was a notable elevation of $\mathrm{R} 2{ }^{*}$ at $1 \mathrm{~h}$ after the second injection of iodixanol observed among all renal areas in Group 2. In CIAKI-rats, BOLD MRI showed that ISOM demonstrated the most sensitive responses to repeated iodixanol administration. This was further confirmed by HIF- $1 \alpha$ immunohistochemical staining findings. Previous studies on ISOM region revealed its sensitivity to ischemic injury $[34,35]$. One possible explanation is that iodixanol diverts medullary blood flow to increase cortical blood flow and results in the deterioration of medullary perfusion and the reduction of renal oxygen tensions [36]. On the other hand, hypoxia was observed to correlate with tubular injury as well [37]. NGAL, as a viable tubular damage marker, is rapidly and massively generated in tubule cells of the kidney after renal ischemia reperfusion, including those of ISOM, released into the urine within hours [38]. Our findings showed that uNGAL was significantly detected within 5 days 


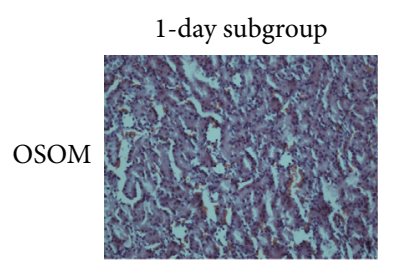

(a)

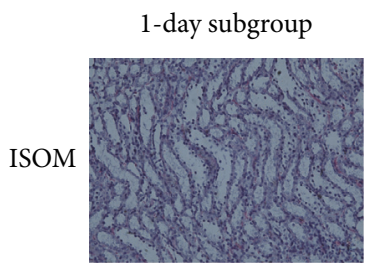

(f)

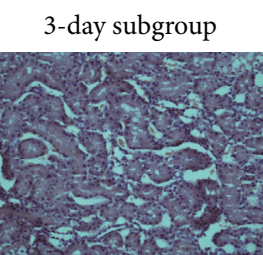

(b)

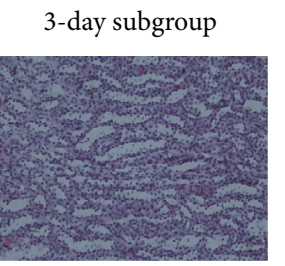

(g) 5-day subgroup

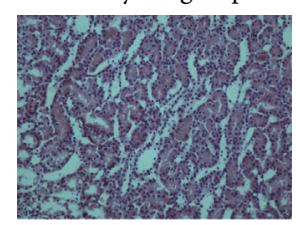

(c)

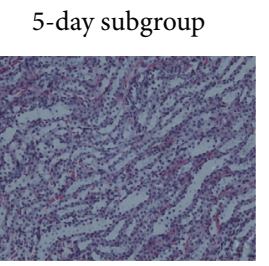

(h)

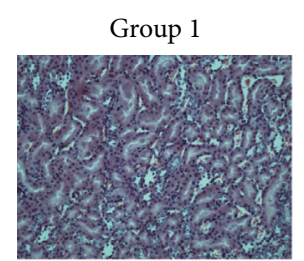

(d)

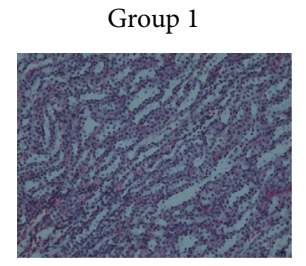

(i)

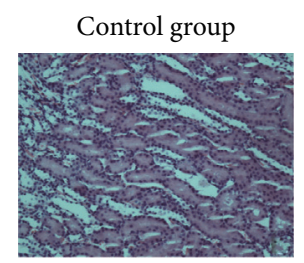

(e)

Control group

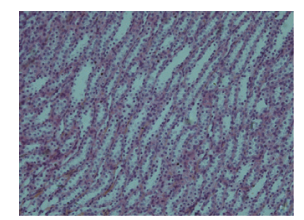

(j)

OSOM
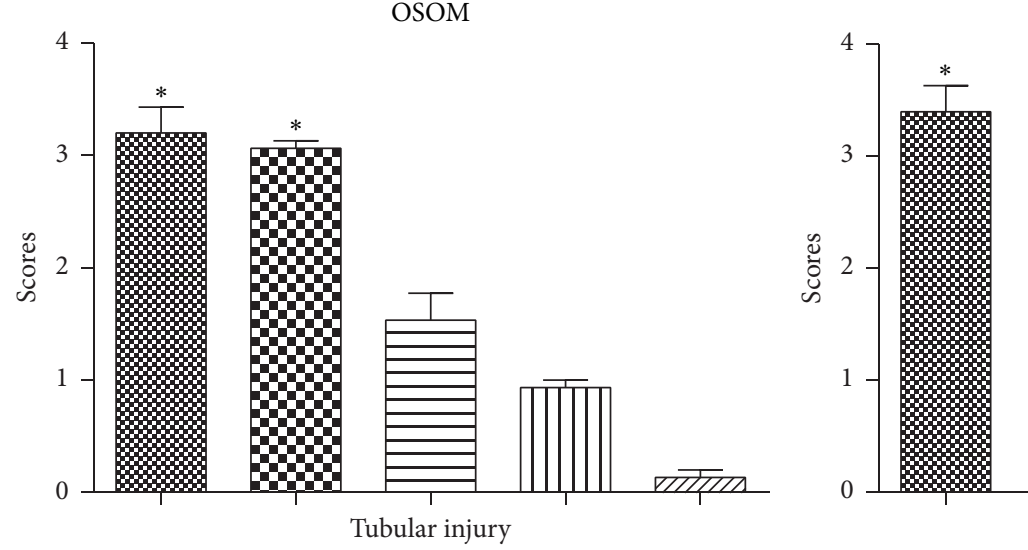

ISOM

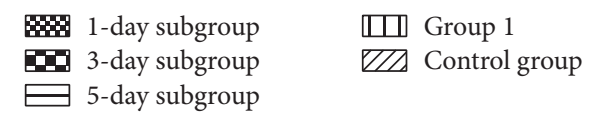

FIGURE 7: Hematoxylin-Eosin (H\&E) stained histological sections of the kidneys in the outer medulla (OSOM and ISOM) at 1h in different groups. Remarkable changes (swollen, broken down, necrotic, and intraluminal desquamation) were observed in part of proximal tubular and distal convoluted tubular epithelial cells in the OSOM and medullary thick ascending limbs, and medullary collecting ducts in the ISOM. Scale bar indicates $100 \mu \mathrm{m}$. Asterisk indicates $P<0.05$ (compared with Group 1).

after the injection of iodixanol, indicating the existence of renal tubular acute injury. In addition, the data presented here are warranted to correlate the observed BOLD MRI changes with both HIF-1 $\alpha$ expression and histopathological changes in the medulla. Therefore, an increase in $\mathrm{R} 2{ }^{*}$ values in ISOM could contribute to determining the risk for subsequent development of CIAKI as evaluated by NGAL [39].

In the current study, the influence of iodixanol on renal water diffusion was evaluated dynamically during the 10 days after the second injection. The prominent decrease of ADC in iodixanol-treated rat groups is aligned with a previous study [40]. One possible explanation of such observation is that the vacuolization found in the cortex may have increased the ratio of cytotoxic edema. Secondly, there was a reduction of renal blood flow that could in return cause longer-lasting iodixanol retention. More importantly, the third contributor could be the changes of tubular fluid resulting from the influenced viscosity of iodixanol, which in return reduced glomerular filtration. Yet, several previous studies suggested a positive linear correlation between glomerular filtration (GFR) and the renal ADC [20, 41].

Similarly, this phenomenon of long-term renal injury caused by iodixanol was reported in clinical routine [42]. It was previously showed that some cases of CIAKI possessed a higher risk to develop a persistent renal damage which regressed to a level close to baseline after over 3 months of time [43]. It was also indicated that AKI might have increased the risk for chronic kidney disease and end-stage renal disease [44]. Thus, CIAKI is not a transient, but a direct cause of aggravated renal functions [45]. Nevertheless, our results confirmed the existence of correlations between CIAKI and longer-lasting renal hypoxia indicated by BOLD findings following repetitive $\mathrm{CM}$ injections. The reason may due to the fact that kidney damage caused by the first injection made the subjects more vulnerable to acute kidney injury 

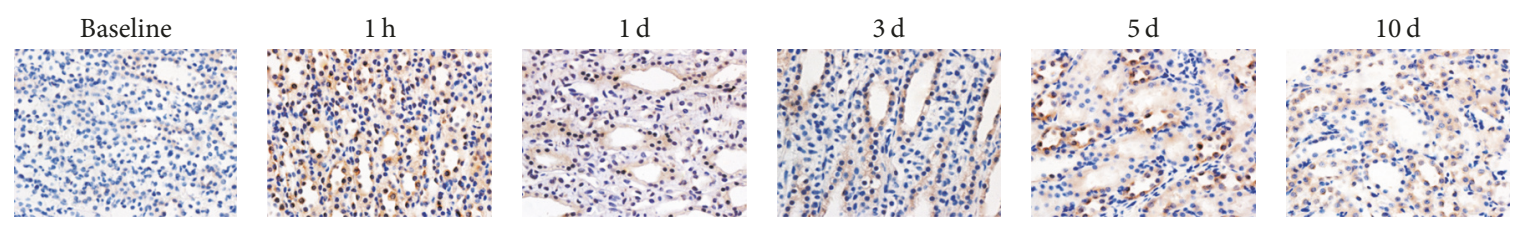

(a)
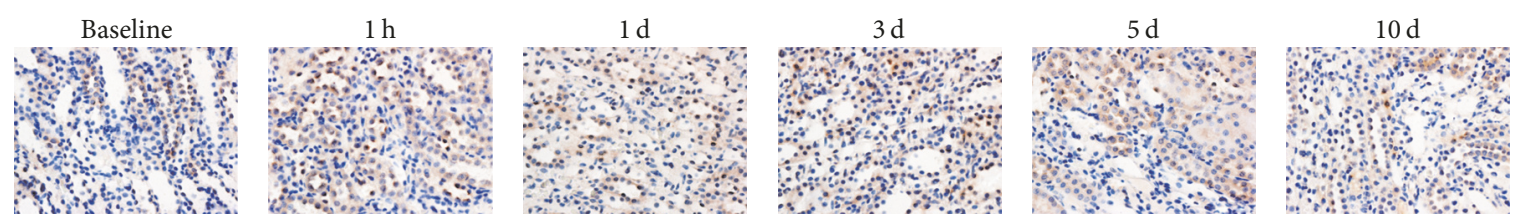

(b)
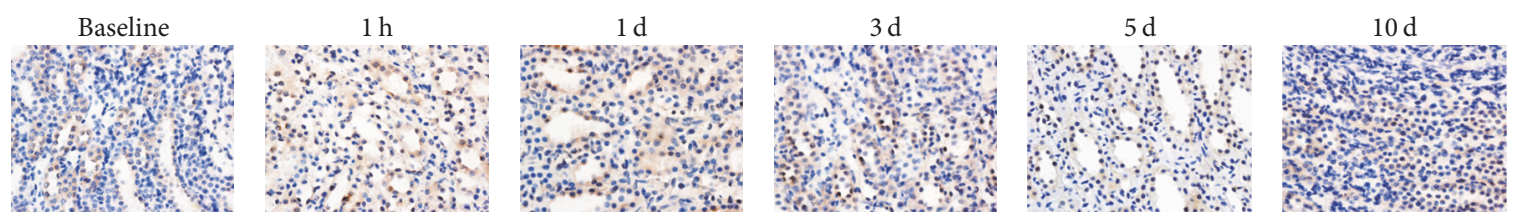

(c)
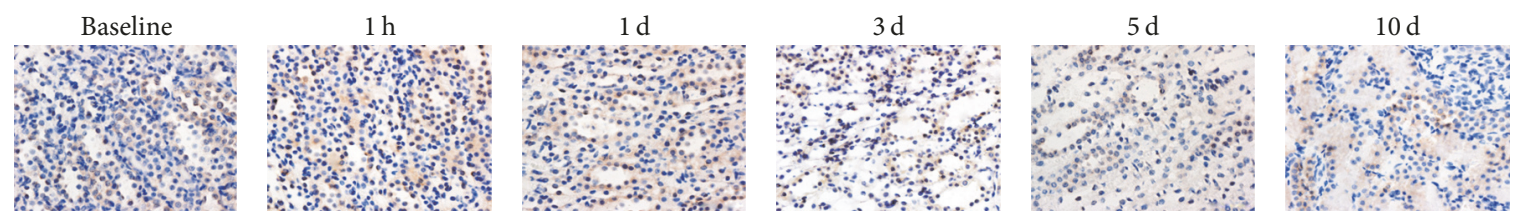

(d)

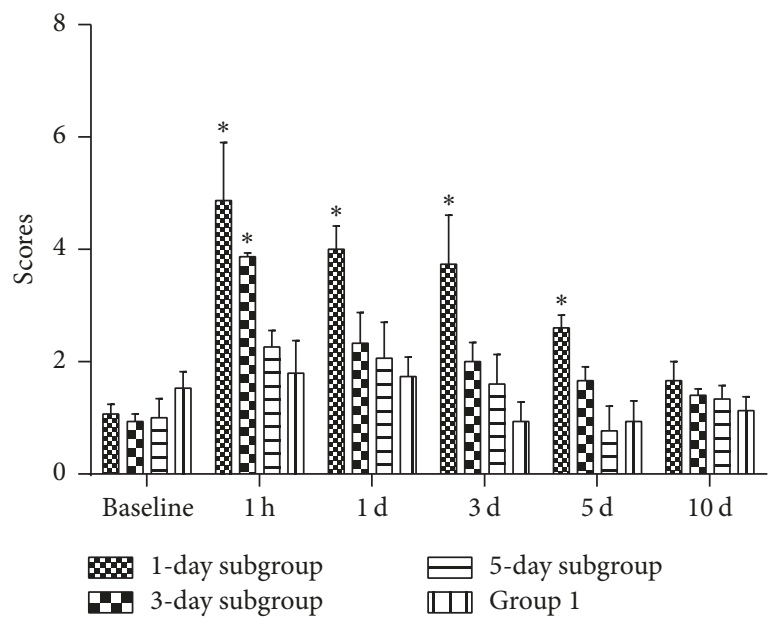

(e)

FIGURE 8: HIF-1 $\alpha$ signals changes in ISOM renal zones. HIF- $1 \alpha$ was mainly localized in the renal ISOM, especially at $1 \mathrm{~h}$. Marked nuclear accumulation of HIF-1 $\alpha$ appears in the 1-day subgroup. Thereafter, staining gradually decreased over time, and they were detectable for 5 days. Asterisk indicates $P<0.05$ (compared with Group 1). Scale bar, $50 \mu \mathrm{m}$. (a) 1-day subgroup; (b) 3-day subgroup; (c) 5-day subgroup; (d) Group 1; (e) HIF-1 $\alpha$ score.

that could be induced by further injections. Our observations were consistent with clinical report that repetitive iodixanol injections faced higher-risk recurrent episodes of kidney injury and a long-term loss of kidney function. It was shown that one-third of patients with AKI during their initial hospitalization experienced repeated episodes of AKI, while each AKI episode doubled the danger of progressive chronic kidney disease [46].
In this study, a comprehensive method used $\mathrm{sCr}$ and uNGAL to evaluate renal damage after CM repeated injection. The results showed that the peak in UNGAL occurred in approximate 1 day, while $\mathrm{sCr}$ occurred in approximate 3 days, which implied that the changes in uNGAL were much earlier than those of blood sCr. This indicated that the levels of urinary NGAL may be useful biomarker for predicting renal prognosis of AKI. 


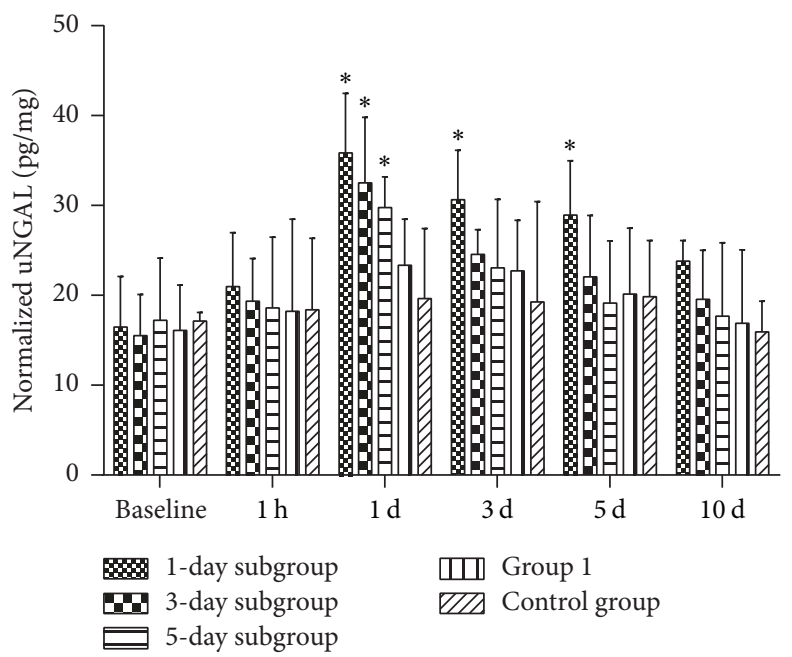

FIGURE 9: The normalized $u$ NGAL $(p g / m g)$ at different time points during the course of contrast injection $(n=3)$. There was a significant increase in uNGAL levels in rats at 1 day, 3 days, and 5 days in 1-day subgroup. Asterisk indicates $P<0.05$ (compared with baseline).

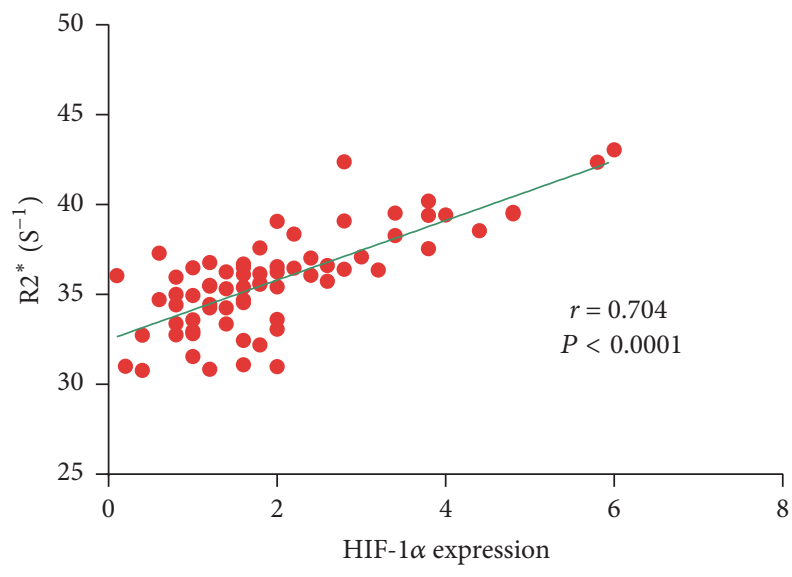

(a)

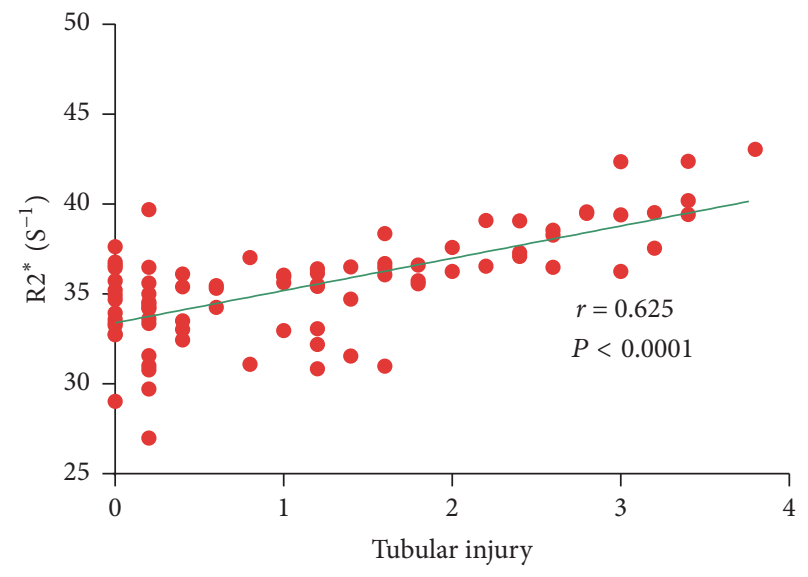

(b)

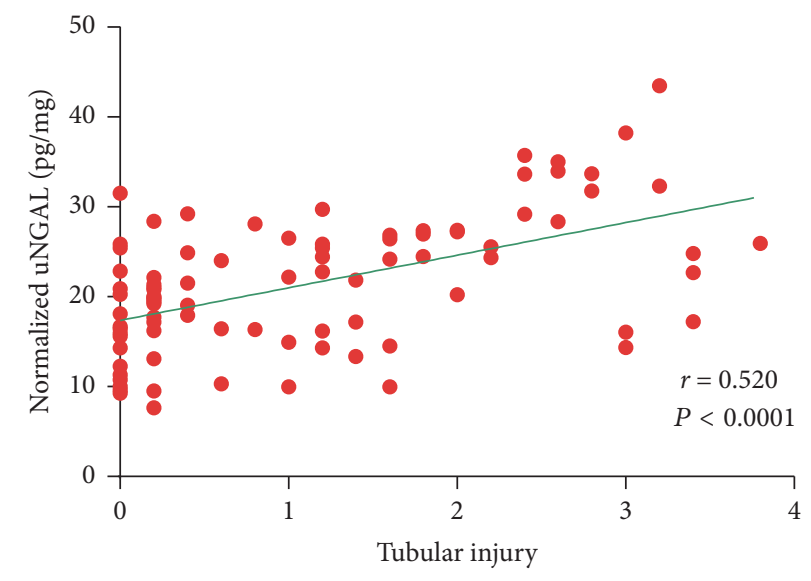

(c)

FIGURE 10: Correlation of $R 2^{*}$ with HIF-1 $\alpha$ expression/tubular injury and tubular injury with $u$ NGAL. (a) The correlation between the measured R2* values and HIF- $1 \alpha$ expression score in the ISOM. (b) The correlation between the measured R2* values and tubular injury score in ISOM. (c) The correlation between all the measured uNGAL and tubular injury score in ISOM. 
Notwithstanding, BOLD MRI can monitor renal oxygenation level affected by CMs administration; however, recent (pre-) clinical studies received the question as to the limitations of renal BOLD MRI [47]. In Seeliger et al.s study, compared with baseline levels, cortical T2* dropped slightly by about $10 \%$ and cortical pO2 reduced by about $40 \%$ [48]. The discrepancy between $\mathrm{T}^{*}$ and $\mathrm{pO} 2$ response to hypoxia is partially due to the decrease of vascular volume fraction. One reason is that CM-induced high tubular fluid viscosity results in an increase in the intratubular pressure. Another major cause is the vasoconstriction after the administration of $\mathrm{CM}$. Additionally, CM can leftward shift the oxyhaemoglobin dissociation curve, so that release of $\mathrm{O} 2$ from haemoglobin $(\mathrm{Hb})$ is impeded. Thus, $\mathrm{T} 2^{*}$ may not accurately reflect blood oxygenation, and we will continue to work on improving the accuracy of the measurement and the quality of images.

\section{Limitations}

Iodixanol was injected at a dose of $4 \mathrm{~g}$ iodine $/ \mathrm{kg}$ body weight in line with previous studies $[29,49]$. The applied dose was based on the body surface area (rat: human $=6: 1$ ) [50], which mimics the dosage used for humans during contrast medium-enhanced CT $(0.5-0.8 \mathrm{~g} \mathrm{I} / \mathrm{kg} \mathrm{BW})$. However, there are a few limitations to be mentioned. First of all, a single dose of CM was applied for each injection. Further research can explore how differences in dosage of repetitive iodixanol will affect the renal functions. Secondly, other risk factors for CIAKI were not considered, such as diabetic nephropathy or chronic renal insufficiency. Additionally, iodixanol was selected as a representative of CMs applied in our study, while further study can compare different CMs with respect to different time intervals. It will be valuable to observe renal damage and to characterize the cause of hypoxia after multiple injections of CMs.

\section{Conclusions}

In conclusion, BOLD and DWI MRI can be applied to examine CIAKI in clinical practice. HIF- $1 \alpha$ and BOLD imaging can verify the hypoxia tissues from different perspectives in CIAKI. Repetitive injection of iodixanol within a short period of time not only induced more pronounced and longerlasting renal hypoxia in the whole kidneys, but also enhanced the structure and functions of kidneys as well as elevated urinary biomarkers.

\section{Conflicts of Interest}

The authors declare that they have no conflicts of interest.

\section{Acknowledgments}

The subject was supported by the National Natural Science Foundation of China (Grant 81571635) and Shenyang Science and Technology Program (Grant F15-199-1-40). The authors thank Zixuan Li for technical assistance (Key Laboratory of Imaging Diagnosis and Interventional Radiology of Liaoning Province, Shenyang, Liaoning, China). Also, they thank
ISMRM 2017 Annual Meeting for giving them the opportunity to present their research and to gain insightful advice which helps them to perfect the paper further.

\section{References}

[1] T. Frenzel, R. Lawaczeck, M. Taupitz et al., "Contrast media for $\mathrm{X}$-ray and magnetic resonance imaging: Development, current status and future perspectives," Investigative Radiology, vol. 50, no. 9, pp. 671-678, 2015.

[2] F. Stacul, A. J. van der Molen, P. Reimer et al., "Contrast induced nephropathy: updated ESUR Contrast Media Safety Committee guidelines," European Radiology, vol. 21, no. 12, pp. 2527-2541, 2011.

[3] K. S. Leow, Y. W. Wu, and C. H. Tan, "Renal-related adverse effects of intravenous contrast media in computed tomography," Singapore Medical Journal, vol. 56, no. 4, pp. 186-193, 2015.

[4] P. A. McCullough, "Contrast-induced acute kidney injury," Journal of the American College of Cardiology, vol. 51, no. 15, pp. 14191428,2008

[5] C. Briguori, E. Donnarumma, C. Quintavalle, D. Fiore, and G. Condorelli, "Contrast-induced acute kidney injury: Potential new strategies," Current Opinion in Nephrology and Hypertension, vol. 24, no. 2, pp. 145-153, 2015.

[6] T. Konno, R. Nakano, R. Mamiya et al., "Expression and Function of Interleukin-1 $\beta$-Induced Neutrophil GelatinaseAssociated Lipocalin in Renal Tubular Cells," PLoS ONE, vol. 11, no. 11, p. e0166707, 2016.

[7] R. Hirsch, C. Dent, H. Pfriem et al., "NGAL is an early predictive biomarker of contrast-induced nephropathy in children," Pediatric Nephrology, vol. 22, no. 12, pp. 2089-2095, 2007.

[8] L.-P. Li, J. Lu, Y. Zhou et al., "Evaluation of intrarenal oxygenation in iodinated contrast-induced acute kidney injury-susceptible rats by blood oxygen level-dependent magnetic resonance imaging," Investigative Radiology, vol. 49, no. 6, pp. 403410, 2014.

[9] A. Lacquaniti, F. Buemi, R. Lupica et al., "Can neutrophil gelatinase-associated lipocalin help depict early contrast material-induced nephropathy?" Radiology, vol. 267, no. 1, pp. 8693, 2013.

[10] S. N. Heyman, S. Rosen, and C. Rosenberger, "Renal parenchymal hypoxia, hypoxia adaptation, and the pathogenesis of radiocontrast nephropathy," Clinical Journal of the American Society of Nephrology, vol. 3, no. 1, pp. 288-296, 2008.

[11] Z. Z. Liu, K. Schmerbach, Y. Lu et al., "Iodinated contrast media cause direct tubular cell damage, leading to oxidative stress, low nitric oxide, and impairment of tubuloglomerular feedback," American Journal of Physiology-Renal Physiology, vol. 306, no. 8, pp. F864-F872, 2014.

[12] D. A. Lauver, E. G. Carey, I. L. Bergin, B. R. Lucchesi, and H. S. Gurm, "Sildenafil citrate for prophylaxis of nephropathy in an animal model of contrast-induced acute kidney injury," PLoS ONE, vol. 9, no. 11, Article ID el13598, 2014.

[13] E. Seeliger, B. Flemming, T. Wronski et al., "Viscosity of contrast media perturbs renal hemodynamics," Journal of the American Society of Nephrology, vol. 18, no. 11, pp. 2912-2920, 2007.

[14] K. Hueper, D. Hartung, M. Gutberlet et al., "Assessment of impaired vascular reactivity in a rat model of diabetic nephropathy: Effect of nitric oxide synthesis inhibition on intrarenal diffusion and oxygenation measured by magnetic resonance imaging," American Journal of Physiology-Renal Physiology, vol. 305, no. 10, pp. F1428-F1435, 2013. 
[15] P. V. Prasad, "Functional MRI of the kidney: Tools for translational studies of pathophysiology of renal disease," American Journal of Physiology-Renal Physiology, vol. 290, no. 5, pp. F958F974, 2006.

[16] L.-P. Li, T. Franklin, H. Du et al., "Intrarenal oxygenation by blood oxygenation level-dependent MRI in contrast nephropathy model: Effect of the viscosity and dose," Journal of Magnetic Resonance Imaging, vol. 36, no. 5, pp. 1162-1167, 2012.

[17] J.-H. Wang, K. Ren, W.-G. Sun, L. Zhao, H.-S. Zhong, and K. Xu, "Effects of iodinated contrast agents on renal oxygenation level determined by blood oxygenation level dependent magnetic resonance imaging in rabbit models of type 1 and type 2 diabetic nephropathy," BMC Nephrology, vol. 15, no. 1, article 140, 2014.

[18] M. Pedersen, T. H. Dissing, J. Morkenborg et al., "Validation of quantitative BOLD MRI measurements in kidney: application to unilateral ureteral obstruction," Kidney International, vol. 67, no. 6, pp. 2305-2312, 2005.

[19] P.-H. Vivier, P. Storey, H. Chandarana et al., "Renal blood oxygenation level-dependent imaging: Contribution of R2 to R2* values," Investigative Radiology, vol. 48, no. 7, pp. 501-508, 2013.

[20] G. Jost, D. C. Lenhard, M. A. Sieber, P. Lengsfeld, J. Hütter, and H. Pietsch, "Changes of renal water diffusion coefficient after application of iodinated contrast agents: Effect of viscosity," Investigative Radiology, vol. 46, no. 12, pp. 796-800, 2011.

[21] S. Haneder, J. Augustin, G. Jost et al., "Impact of iso- and lowosmolar iodinated contrast agents on BOLD and diffusion MRI in swine kidneys," Investigative Radiology, vol. 47, no. 5, pp. 299305, 2012.

[22] Y.-C. Wang, A. Tang, D. Chang, S.-J. Zhang, and S. Ju, "Significant perturbation in renal functional magnetic resonance imaging parameters and contrast retention for iodixanol compared with iopromide: An experimental study using blood-oxygenlevel/dependent/diffusion-weighted magnetic resonance imaging and computed tomography in rats," Investigative Radiology, vol. 49, no. 11, pp. 699-706, 2014.

[23] W. B. Chen, L. Liang, B. Zhang et al., "To evaluate the damage of renal function in CIAKI rats at 3T: using ASL and BOLD MRI," BioMed Research International, vol. 2015, Article ID 593060, 8 pages, 2015.

[24] L.-P. Li, J. Lu, T. Franklin, Y. Zhou, R. Solomon, and P. V. Prasad, "Effect of iodinated contrast medium in diabetic rat kidneys as evaluated by blood-oxygenation-level-dependent magnetic resonance imaging and urinary neutrophil gelatinaseassociated lipocalin," Investigative Radiology, vol. 50, no. 6, pp. 392-396, 2015.

[25] L. Liang, W. B. Chen, K. W. Chan et al., "Using intravoxel incoherent motion MR imaging to study the renal pathophysiological process of contrast-induced acute kidney injury in rats: comparison with conventional DWI and arterial spin labelling," European Radiology, 2015.

[26] M. Colbay, S. Yuksel, I. Uslan, and et al., "Novel approach for the prevention of contrast nephropathy," Experimental and Toxicologic Pathology Official Journal of the Gesellschaft Für Toxikologische Pathologie, vol. 62, no. 1, pp. 81-89, 2010.

[27] K. Manotham, T. Tanaka, T. Ohse et al., "A biologic role of HIF1 in the renal medulla," Kidney International, vol. 67, no. 4, pp. 1428-1439, 2005.

[28] C. Xin, X. Yulong, C. Yu, C. Changchun, Z. Feng, and M. Xinwei, "Urine neutrophil gelatinase-associated lipocalin and interleukin-18 predict acute kidney injury after cardiac surgery," Renal Failure, vol. 30, no. 9, pp. 904-913, 2008.
[29] D. C. Lenhard, A.-L. Frisk, P. Lengsfeld, H. Pietsch, and G. Jost, "The effect of iodinated contrast agent properties on renal kinetics and oxygenation," Investigative Radiology, vol. 48, no. 4, pp. 175-182, 2013.

[30] D. N. Cruz, M. A. Perazella, R. Bellomo et al., "Extracorporeal Blood Purification Therapies for Prevention of RadiocontrastInduced Nephropathy: A Systematic Review," American Journal of Kidney Diseases, vol. 48, no. 3, pp. 361-371, 2006.

[31] G. Jost, P. Lengsfeld, D. C. Lenhard, H. Pietsch, J. Hütter, and M. A. Sieber, "Viscosity of iodinated contrast agents during renal excretion," European Journal of Radiology, vol. 80, no. 2, pp. 373377, 2011.

[32] C. Briguori, C. Quintavalle, F. De Micco, and G. Condorelli, "Nephrotoxicity of contrast media and protective effects of acetylcysteine," Archives of Toxicology, vol. 85, no. 3, pp. 165-173, 2011.

[33] N. Alessandri, L. Lanzi, C. M. Garante et al., "Prevention of acute renal failure post-contrast imaging in cardiology: a randomized study," European Review for Medical and Pharmacological Sciences, vol. 17, pp. 13-21, 2013.

[34] K. E. De Greef, D. K. Ysebaert, V. Persy, S. R. Vercauteren, and M. E. De Broe, "ICAM-1 expression and leukocyte accumulation in inner stripe of outer medulla in early phase of ischemic compared to $\mathrm{HgCl} 2$-induced ARF," Kidney International, vol. 63, no. 5, pp. 1697-1707, 2003.

[35] S. N. Heyman, M. Brezis, C. A. Reubinoff et al., "Acute renal failure with selective medullary injury in the rat," The Journal of Clinical Investigation, vol. 82, no. 2, pp. 401-412, 1988.

[36] M. Brezis, S. Rosen, P. Silva, and F. H. Epstein, "Selective vulnerability of the medullary thick ascending limb to anoxia in the isolated perfused rat kidney," The Journal of Clinical Investigation, vol. 73, no. 1, pp. 182-190, 1984.

[37] C. Rosenberger, S. N. Heyman, S. Rosen et al., "Up-regulation of HIF in experimental acute renal failure: Evidence for a protective transcriptional response to hypoxia," Kidney International, vol. 67, no. 2, pp. 531-542, 2005.

[38] G. M. McMahon and S. S. Waikar, "Biomarkers in nephrology: Core curriculum 2013," American Journal of Kidney Diseases, vol. 62, no. 1, pp. 165-178, 2013.

[39] L.-P. Li, J. Thacker, J. Lu et al., "Efficacy of preventive interventions for iodinated contrast-induced acute kidney injury evaluated by intrarenal oxygenation as an early marker," Investigative Radiology, vol. 49, no. 10, pp. 647-652, 2014.

[40] J.-P. Laissy, D. Menegazzo, E. Dumont et al., "Hemodynamic effect of iodinated high-viscosity contrast medium in the rat kidney: A diffusion-weighted MRI feasibility study," Investigative Radiology, vol. 35, no. 11, pp. 647-652, 2000.

[41] R. Toya, S. Naganawa, H. Kawai, and M. Ikeda, "Correlation between estimated glomerular filtration rate (eGFR) and apparent diffusion coeffcient (ADC) values of the kidneys," Magnetic Resonance in Medical Sciences, vol. 9, no. 2, pp. 59-64, 2010.

[42] S.-H. Chou, Z. J. Wang, J. Kuo et al., "Persistent renal enhancement after intra-arterial versus intravenous iodixanol administration," European Journal of Radiology, vol. 80, no. 2, pp. 378386, 2011.

[43] M. Maioli, A. Toso, M. Leoncini, M. Gallopin, N. Musilli, and F. Bellandi, "Persistent renal damage after contrast-induced acute kidney injury: incidence, evolution, risk factors, and prognosis," Circulation, vol. 125, no. 25, pp. 3099-3107, 2012.

[44] S. G. Coca, S. Singanamala, and C. R. Parikh, "Chronic kidney disease after acute kidney injury: a systematic review and metaanalysis," Kidney International, vol. 81, no. 5, pp. 442-448, 2012. 
[45] J. Zhong, H.-C. Yang, and A. B. Fogo, "A perspective on chronic kidney disease progression," American Journal of PhysiologyRenal Physiology, vol. 312, no. 3, pp. F375-F384, 2017.

[46] C. V. Thakar, A. Christianson, J. Himmelfarb, and A. C. Leonard, "Acute kidney injury episodes and chronic kidney disease risk in diabetes mellitus," Clinical Journal of the American Society of Nephrology, vol. 6, no. 11, pp. 2567-2572, 2011.

[47] T. Niendorf, A. Pohlmann, K. Arakelyan et al., "How bold is blood oxygenation level-dependent (BOLD) magnetic resonance imaging of the kidney? Opportunities, challenges and future directions," Acta physiologica (Oxford, England), vol. 213, no. 1, pp. 19-38, 2015.

[48] E. Seeliger, K. Cantow, K. Arakelyan, M. Ladwig, P. B. Persson, and B. Flemming, "Low-dose nitrite alleviates early effects of an X-ray contrast medium on renal hemodynamics and oxygenation in rats," Investigative Radiology, vol. 49, no. 2, pp. 7077, 2014.

[49] D. C. Lenhard, H. Pietsch, M. A. Sieber et al., "The osmolality of nonionic, iodinated contrast agents as an important factor for renal safety," Investigative Radiology, vol. 47, no. 9, pp. 503-510, 2012.

[50] "U.S. Department of Health and Human Services. Food and Drug Administration. Center for Drug Evaluation and Research: Guidance for Industry: Estimating the maximum safe starting dose in initial clinical trials for therapeutics in adult healthy volunteers, 2005," http://www.fda.gov/downloads/ Drugs/GuidanceComplianceRegulatoryInformation/Guidances/ ucm078932.pdf. 


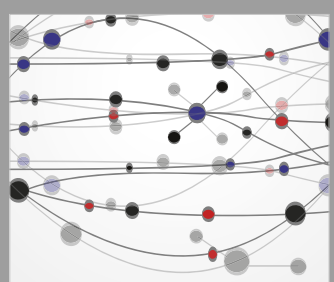

The Scientific World Journal
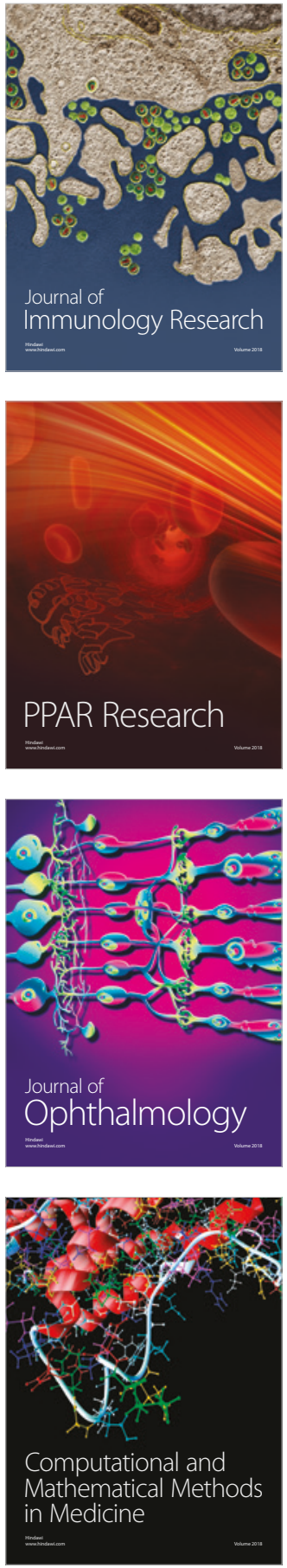

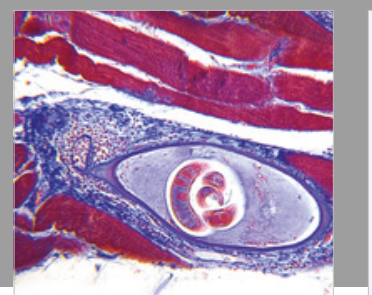

Gastroenterology Research and Practice

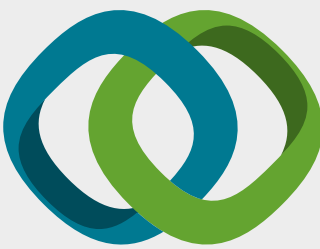

\section{Hindawi}

Submit your manuscripts at

www.hindawi.com
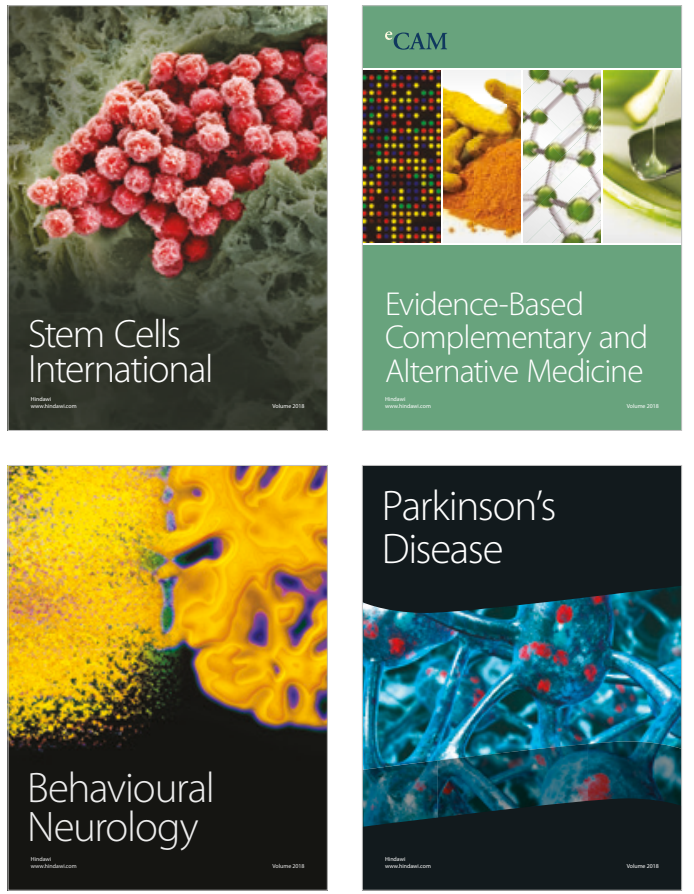

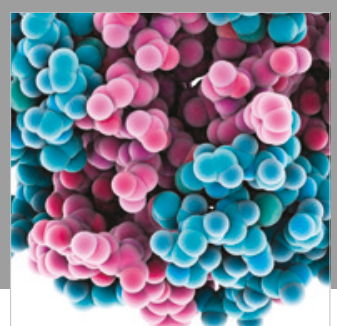

ournal of

Diabetes Research

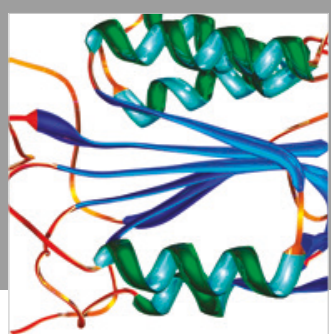

Disease Markers
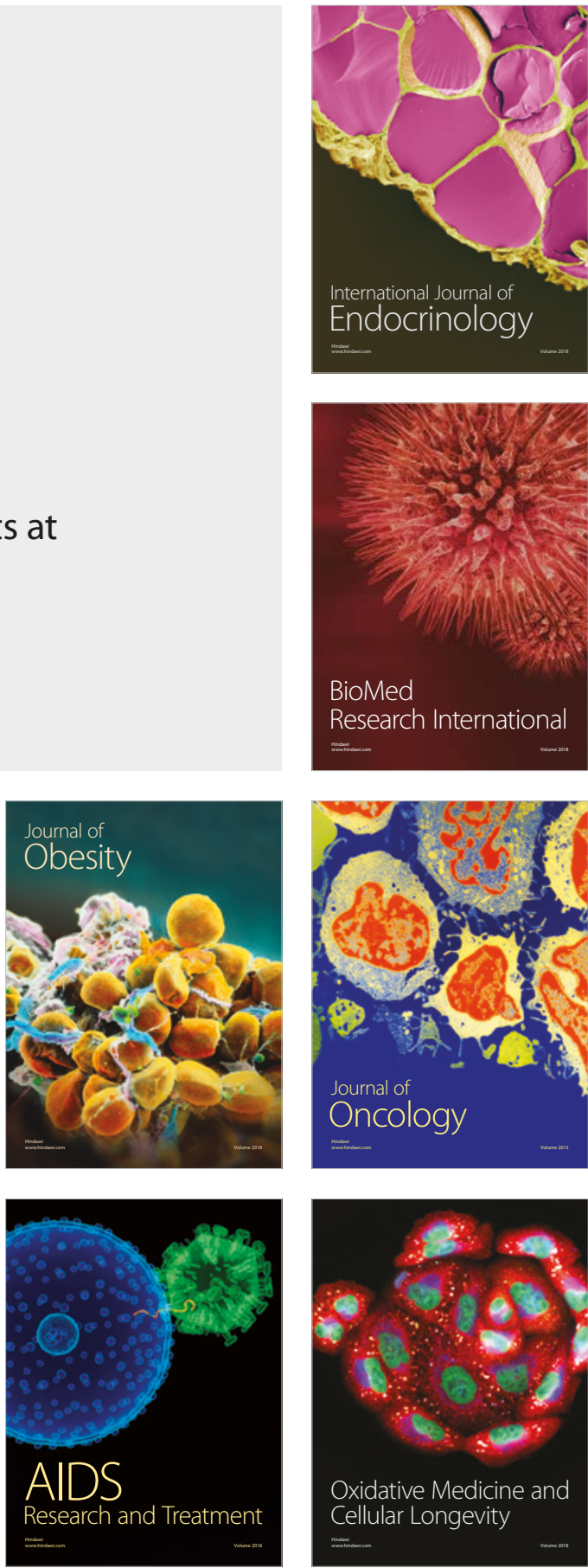